\title{
Hybrid Cognitive-Radio NOMA with Blind Transmission Mode Identification and BER Constraints
}

\author{
Hamad Yahya, Graduate Student Member, IEEE, Emad Alsusa Senior Member, IEEE, \\ and Arafat Al-Dweik, Senior Member, IEEE,
}

\begin{abstract}
The synergy of nonorthogonal multiple access (NOMA) and cognitive radio (CR) can provide efficient spectrum utilization for future wireless networks and enable supporting heterogeneous quality of service $(\mathrm{QoS})$ requirements. In this context, this article aims at evaluating the throughput of a downlink CR-NOMA network where the secondary user (SU) data is opportunistically multiplexed with the primary user (PU) data using power-domain NOMA. The data multiplexing process is constrained by the PU QoS requirements. The multiplexing process can be considered seamless with respect to the PU because its receiver design will generally remain unchanged. Moreover, we consider the case where the SU detects its own data by blindly identifying the adopted transmission mode (TM) at the base station, which can be PU orthogonal multiple accesS PU-OMA, SU-OMA, PU/SU-NOMA, and no transmission. Consequently, the network can be classified as a hybrid underlayinterweave. The detection process is considered blind because the SU does not receive side information about the adopted TM. The obtained analytical results corroborated by Monte Carlo simulation results show that the proposed CR-NOMA network can provide substantial throughput improvement over conventional NOMA networks, particularly at low signal-to-noise ratios (SNRs) because the unutilized PU spectrum can be used by the SU. Moreover, in good channel conditions the PU can tolerate some interference from the $\mathrm{SU}$, which may improve the channel utilization significantly.
\end{abstract}

Index Terms-Cognitive radio inspired nonorthogonal multiple access (CR-NOMA), hybrid multiple access, blind cognitive receiver, classifier, counting rules, throughput.

\section{INTRODUCTION}

$\mathbf{N}$ ONORTHOGONAL multiple access (NOMA) is a promising candidate for future radio networks due to its ability to improve the connectivity and provide high spectral efficiency [1]. It can also support diverse quality-of-service (QoS) requirements when users with distinct channel conditions are paired to transmit over the same transmission resources. In power-domain NOMA, the users share the same transmission time-slot or frequency-band, while the multiplexing is performed by assigning each user a different power level. The assigned power value might be allocated based on the users'

Hamad Yahya and Emad Alsusa are with the Department of Electrical and Electronic Engineering, The University of Manchester, Manchester, M13 9PL, UK. (email: hamad.mohamadaliyahya@manchester.ac.uk, e.alsusa@manchester.ac.uk)

Arafat Al-Dweik is with Center for Cyber Physical Systems (C2PS), Khalifa University, Abu Dhabi, P.O.Box 127788, UAE. He is also with the Department of Electrical and Computer Engineering, Western University, London, ON, Canada. (e-mail: dweik@fulbrightmail.org). channel conditions QoS requirements. At the receiver side, successive interference cancellation (SIC) can be used to separate the signal of each user and eliminate the interference of other users. The gained spectral efficiency of NOMA typically comes at the expense of bit error rate (BER) degradation, which is not the case for orthogonal multiple access (OMA) techniques due to the inter-user interference (IUI) effect in NOMA [2]-[4].

Cognitive radio $(\mathrm{CR})$, is another prominent technology that can be used to combat the inefficient spectrum utilization problem [5]-[7]. The main principle of CR is to allow unlicensed users, called secondary users (SUs) or cognitive users, to opportunistically or collaboratively share the licensed users' spectrum, called primary users (PUs). The spectrum sharing process should be constrained by the PU QoS requirements [8]. $\mathrm{CR}$ has three main types which are interweave $\mathrm{CR}$, underlay $\mathrm{CR}$ and overlay $\mathrm{CR}$. In interweave $\mathrm{CR}$, the secondary transmitter transmits to the secondary receivers when the spectrum is not occupied by PU. This requires certain activity detection technology, such as spectrum sensing, to identify idle spectrum bands. In underlay $\mathrm{CR}$, the PU and SU transmit simultaneously given that the SU interference is tolerable by the PU. In overlay $\mathrm{CR}$, the SU collaborates with the PU to relay its messages. Therefore, it can be noted that spectrum sharing is the common principle between NOMA and CR, and also the objectives of both technologies are aligned, which triggered several research attempts to combine NOMA and CR technologies to improve the spectrum sharing process. The main research work that considered the integration of CR and NOMA is summarized in the following subsection.

\section{A. Related Work}

Because CR and NOMA technologies typically introduce interference, integrating them should be performed while considering the performance degradation and QoS for the involved users [5], [9]. For example, four schemes are proposed in [9], which are the interweave cognitive NOMA, underlay cognitive NOMA, overlay cognitive NOMA and cognitive radio inspired NOMA (CR-NOMA). The first three are similar to the conventional CR, except that NOMA is employed for the secondary network users. However, CR-NOMA is based on a single transmitter that prioritizes the users in the network and tries to guarantee the QoS requirements for a single or multiple users through a unique power assignment policy that gives higher priority to the PU while serving the SU opportunistically. Also, 
a cooperative framework is introduced to improve the system performance [9].

Underlay cognitive NOMA is studied in [10], [11] where the authors in [10] apply the concept to a large scale network of randomly deployed users modeled by stochastic geometry. Closed-form outage probability is derived while considering two power constraints at the primary transmitter. The first is fixed power constraint and the second ensures proportionality to the secondary transmitter power. Also, fixed power coefficients are considered for NOMA users. It is shown that underlay cognitive NOMA outperforms underlay cognitive OMA if power allocation and rate requirements are selected carefully. Similarly, Arzykulov et al. [11] proposed cooperative relaying for the secondary network that employs a decode-and-forward half duplex relay. The power coefficients are optimized based on the channel quality to achieve fairness among users in term of outage probability. Also, imperfect channel state information (CSI) is assumed and the outage-based throughput is evaluated. Moreover, overlay cognitive NOMA network with imperfect SIC is investigated in [12], where asymptotic expressions for outage and throughput are derived, and the optimal power allocation that maximizes the system throughput is computed.

The CR-NOMA proposed by Ding et al. [13] has a power assignment policy that is designed to strictly satisfy the PU rate QoS requirement. The outage probability is derived and compared to the fixed power NOMA. Interestingly, it is shown that fixed power NOMA tends to pair users with the most distinct channel conditions. However, CR-NOMA rends to pair the best channel user with the second best channel user. It is worth noting that the channel gains follow the order statistics model, and hence, the instantaneous channel gain of the near user is consistently higher than that of the far user. Moreover, it is shown that the SU diversity gain is limited by the PU channel quality. Hence, a dynamic hybrid NOMA scheme for the downlink is proposed in [14] to overcome the diversity gain limitation, provide more balanced trade-off between the users' rate QoS, and avoid an outage probability of one as in fixed power NOMA. This is achieved by adapting the power coefficients based on the instantaneous channel conditions. Moreover, the power allocation scheme is generalized to the downlink and uplink in [15].

The concept of CR-NOMA is extended to multiple-inputmultiple-output (MIMO) systems [16], where a precoder is designed to distinguish between the users' channels. Two power assignment policies are set to ensure the rate QoS requirement of the degraded user instantaneously or over the long term. While CR-NOMA is studied for unicast transmissions in [13][16], it is also considered for mixed multicast and unicast transmissions in [17]-[19]. Two power assignment policies are proposed in [17], one to satisfy the rate QoS requirements of the multicast users, and the other is to satisfy the rate QoS requirements of the unicast user. Cooperative multicast CRNOMA is investigated in [19] where secondary multicast users act as a relays to improve the performance of the primary and secondary networks. The proposed scheduling strategies require knowledge of CSI at the transmitter side. The authors introduce a new performance metric known as the mutual outage, which indicates the probability that any of the users is in outage.
The concept of hybrid NOMA/OMA (H-NOMA) is similar to CR-NOMA, however, it is emphasizes more on the matter of dynamic multiple access [20]-[27]. The authors of [20] propose an an H-NOMA system for downlink multiple-inputsingle-output (MISO) channels, where the base station uses zero-forcing spatial multiplexing precoding if the channel is not quasi-degraded, while NOMA precoding is used if the channel is quasi-degraded. This hybrid precoding scheme imposes the requirement of feedback to inform the receivers about the selected precoding scheme. Moreover, energy efficient power allocation is investigated in [21] for downlink H-NOMA systems where an optimal and a heuristic power allocation algorithms are proposed. The authors in [22] investigate the throughput of H-NOMA for visible light communications where it is shown that H-NOMA achieves better throughput and fairness when compared to static multiple access. In addition, cooperative techniques are employed with H-NOMA to improve the sum rate and reduce outage in [23]-[27]. For example, decode-andforward buffer-aided relays for downlink is proposed in [26] where practical issues such as outdated CSI, packets' retransmission and transmission mode signaling are addressed. HNOMA for the uplink is considered in [25] where fixed and dynamic power allocation are considered, and the switching between the two multiple access schemes is based on CSI and the buffer state.

The practical limitations of H-NOMA such as the signaling overhead are studied in [28]-[31], where blind signal classification methods are proposed. The work in [28] propose a phase rotation-based or pilot-based assisted blind classification where the classifier is a three-step maximum likelihood (ML), which blindly estimates the multiple access technique, modulation orders and power coefficients. The paper studies the effect of classification errors on the system's capacity and signal-tointerference plus noise ratio (SINR). In [29], a feature-extraction based classifier is proposed and the BER performance of the classifier-detector is studied. Also, an ML-based classifier is proposed and analyzed in [30].

\section{B. Motivation and Contribution}

As can noted from the surveyed literature, and to the best of the authors' knowledge, there is no work in the literature that considers uncoordinated downlink CR-NOMA networks with hybrid CR. Particularly when imperfect transmission mode identification and BER constraints are considered for the primary and secondary users. Therefore, we propose in this work a new downlink hybrid CR-NOMA network that guarantees the BER QoS satisfaction the primary and secondary users. The proposed network model is based on combining a hybrid underlay-interweave CR with NOMA to increase the throughput while satisfying the users' BER QoS requirements. To reduce signaling overhead, a blind cognitive receiver is proposed to identify the adopted multiple access technique blindly without side information from the transmitter. The obtained results show that the proposed scheme can significantly increase the network throughput, even when considering imperfect classification and identification at the SU. More specifically, the contribution of this article can be summarized as follows: 


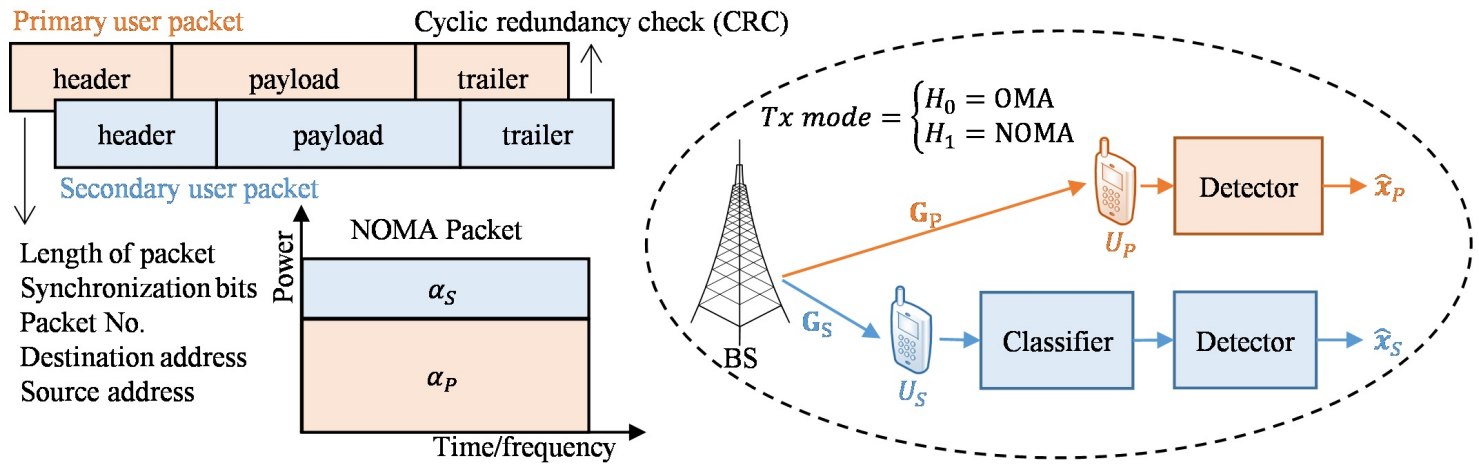

Fig. 1: Illustrative diagram of the downlink CR-NOMA system model.

1) Propose an uncoordinated downlink CR-NOMA network with hybrid CR and users' BER constraints.

2) Analyze the performance of the proposed blind cognitive receiver, where the considered performance metrics are the probability of false alarm and probability of missdetection.

3) Evaluate the performance of various rules to fuse the symbol-based local observations in order to find the global decision on a packet.

4) Derive the system's throughput while considering imperfect detection at the PU and imperfect classification and detection at the SU.

5) Use Monte Carlo simulation to corroborate the derived analytical expressions.

\section{Paper Organization}

The remaining contents of the article are organized as follows. In Sec. II, the system and channel models are introduced. In Sec. III, the performance of multiple access classifier is analyzed, while Sec. IV presents the analysis of the considered decision rules. Then, Sec. V derives the packet error rate (PER), while Sec. VI shows the derivations of the system throughput. Sec. VII presents the analytical and Monte Carlo simulation results, and finally Sec. VIII concludes the article.

\section{Notations}

The notations used throughout the article are as follows. Boldface uppercase and lowercase symbols, such as $\mathbf{X}$ and $\mathbf{x}$ will denote matrices and row/column vectors, respectively. The transpose is denoted by $(\cdot)^{T}$ and the set of complex numbers is denoted by $\mathbb{C} . \operatorname{Pr}(\cdot)$ is the probability of an event, $f(\cdot)$ is the probability density function (PDF) of a random variable, $F(\cdot)$ is cumulative distribution function (CDF) of a random variable, $\mathbb{E}[\cdot]$ is the statistical expectation, $\lceil$.$\rceil is the ceiling operator, |\cdot|$ is the absolute value, $\Re[\cdot]$ and $\Im[\cdot]$ denotes the real and imaginary components, $\left(\begin{array}{l}n \\ k\end{array}\right)$ denotes the binomial coefficients and $\left(\begin{array}{c}n \\ k_{1}, k_{2}, \ldots, k_{N}\end{array}\right)$ denotes the multinomial coefficients. The diagonal matrix with identical elements is represented as $\operatorname{diag}(a)$, where $a$ is the diagonal. The identity $N \times N$ matrix is denoted as $\mathbf{I}_{N}$. The complex Gaussian random variable with a zero mean and $\sigma^{2}$ variance is denoted as $\mathcal{C N}\left(0, \sigma^{2}\right)$. The incomplete upper Gamma function is denoted by $\Gamma(a, x)=\int_{x}^{\infty} \exp (-t) t^{a-1} \mathrm{~d} t$.
TABLE I: Summary of Transmission Modes.

\begin{tabular}{|l||c|l|}
\hline & Packet & \multicolumn{1}{c|}{ Conditions } \\
\hline \hline $\mathrm{TM}_{1}$ & PU-OMA & $\begin{array}{l}\left\{\left\{P_{B_{P}}^{\mathrm{O}} \leq \tau_{P}\right\} \cap\left\{P_{B_{P}}^{\mathrm{N}}>\tau_{P}\right\}\right\} \cup \\
\left\{\left\{P_{B_{P}}^{\mathrm{N}} \leq \tau_{P}\right\} \cap\left\{P_{B_{S}}^{\mathrm{N}}>\tau_{S}\right\}\right\}\end{array}$ \\
\hline $\mathrm{TM}_{2}$ & PU/SU-NOMA & $\left\{P_{B_{P}}^{\mathrm{N}} \leq \tau_{P}\right\} \cap\left\{P_{B_{S}}^{\mathrm{N}} \leq \tau_{S}\right\}$ \\
\hline $\mathrm{TM}_{3}$ & SU-OMA & $\left\{P_{B_{P}}^{\mathrm{O}}>\tau_{P}\right\} \cap\left\{P_{B_{S}}^{\mathrm{O}} \leq \tau_{S}\right\}$ \\
\hline $\mathrm{TM}_{4}$ & Idle & $\left\{P_{B_{P}}^{\mathrm{O}}>\tau_{P}\right\} \cap\left\{P_{B_{S}}^{\mathrm{O}}>\tau_{S}\right\}$ \\
\hline
\end{tabular}

\section{System And Channel Models}

\section{A. System Model}

This work considers the downlink transmission of a packetbased point-to-multi-point CR-NOMA network. Each packet consists of $L$ symbols and the total packet time is assumed to be less than the channel coherence time. The packets' structure follows the conventional structure where each packet has a header that contains fields for synchronization bits, packet number, source and destination addresses. The payload field is dedicated to the user data symbols, and the trailer that is used for error flow control cyclic redundancy check (CRC) or checksum bits. The main system elements are shown in Fig. 1, which include the base station (BS), far and near users. The far user is considered as the PU and denoted by $U_{P}$, while the near user is the $\mathrm{SU}$ and denoted by $U_{S}$. Although the proposed system may accommodate multiple primary and multiple secondary users, the system complexity may increase substantially. Therefore, the number of users per channel is limited to one PU and one SU. The PU is given the highest priority because it is the licensed user. The SU is assumed to be delay-tolerant and opportunistic user. The SU may or may not receive a packet in each transmission slot, such decision is made at the BS. The proposed CRNOMA aims at improving the spectral efficiency of the system while maintaining the BER constraints satisfied for both users. To satisfy the BER constraints, the transmission mode (TM) should be changed adaptively on a packet-by-packet basis while considering the users' BERs $P_{B_{j}}^{m}$ and BERs constraints $\tau_{j}$, where $j \in(P, S)$, i.e., primary or secondary, and $m \in(\mathrm{N}, \mathrm{O})$ where N stands for NOMA while O stands for OMA. Table I summarizes all possible TMs that can be adopted by the BS. It is worth noting that the average BER for the entire packet is equal to the BER for each symbol due to the flat fading effect. The table can be described as follows:

- $\mathrm{TM}_{1}$ : Only PU packet is sent using OMA when OMA 
strictly satisfies PU or if NOMA satisfies PU and NOMA does not satisfy SU.

- $\mathrm{TM}_{2}$ : PU and SU packets are multiplexed and transmitted using NOMA when NOMA can satisfy both users jointly.

- $\mathrm{TM}_{3}$ : Only SU packet is sent using OMA when OMA can only satisfy SU.

- $\mathrm{TM}_{4}$ : No packets are sent if OMA does not satisfy none of the users.

The selection of a particular TM depends on the users' BER constraints, which are affected by the modulation orders, power coefficients and channels' quality. In this work we assume that CSI of all users is available at the BS, which can be achieved through a feedback channel, or using the channel reciprocity concept in time-division duplexing (TDD). However, a fully flexible configuration with adaptive power and modulation orders requires excessive signaling overhead and high complexity transmitter/receiver design [28]-[30], [32], [33]. Therefore, the modulation order in this work is fixed to binary phase shift keying (BPSK) to reflect on the normalized throughput and have a tractable analysis for the cognitive receiver. Moreover, fixed power coefficients are used for the NOMA scenario, which can be estimated in the initialization phase. Although NOMA and OMA BER expressions can be found in the literature [4], they will be stated in the following subsections for the sake of completeness and to unify the notations.

\section{B. Received Signal Model}

The received packet-based signal can be written as

$$
\mathbf{y}_{j}= \begin{cases}\mathbf{G}_{j} \mathbf{x}_{P}+\mathbf{w}_{j}, & \mathrm{TM}_{1} \\ \mathbf{G}_{j}\left(\sqrt{\alpha_{P}} \mathbf{x}_{P}+\sqrt{\alpha_{S}} \mathbf{x}_{S}\right)+\mathbf{w}_{j}, & \mathrm{TM}_{2} \\ \mathbf{G}_{j} \mathbf{x}_{S}+\mathbf{w}_{j}, & \mathrm{TM}_{3} \\ \mathbf{w}_{j}, & \mathrm{TM}_{4}\end{cases}
$$

where $\mathbf{y}_{j} \in \mathbb{C}^{L \times 1}$, and the power coefficients are selected such that $\alpha_{P}+\alpha_{S}=1$. The PU data packet is $\mathbf{x}_{P}=\left[x_{P}^{(1)}, x_{P}^{(2)}, \ldots, x_{P}^{(L)}\right]^{T}$ and the SU packet $\mathbf{x}_{S}=$ $\left[x_{S}^{(1)}, x_{S}^{(2)}, \ldots, x_{S}^{(L)}\right]^{T}$. The additive-white Gaussian noise (AWGN) vector $\mathbf{w}_{j} \sim \mathcal{C N}\left(0, \sigma_{w_{j}}^{2} \mathbf{I}_{L}\right)$ where $\sigma_{w_{j}}^{2}=N_{0}$. The complex channel gain for single-input-single-output (SISO) channel is given $\mathbf{G}_{j} \in \mathbb{C}^{L \times L}$, which corresponds to block Rayleigh fading over one packet interval, that is $\mathbf{G}_{j}=g_{j} \mathbf{I}_{L}$, $g_{j}=\check{g}_{j} \times \Upsilon_{j}^{-\lambda}, \check{g}_{j} \sim \mathcal{C N}\left(0, \sigma_{\check{g}_{j}}^{2}\right), \Upsilon_{j}$ is the distance between the BS and the $j$ th user and $\lambda$ is the pathloss exponent. The small scale channel gains are assumed to be independent and identically distributed (i.i.d). The signal-to-noise-ratio (SNR) is defined as $\mathrm{SNR} \triangleq 1 / N_{0}$ to have a common metric between the users. On the other hand, the instantaneous SNR is $\gamma_{j} \triangleq\left|g_{j}\right|^{2} / \tilde{N}_{0}$ and $\tilde{N}_{0}=0.5 N_{0}$, while the average SNR is $\bar{\gamma}_{j} \triangleq \mathbb{E}\left[\left|g_{j}\right|^{2}\right] / \tilde{N}_{0}$. It is worth noting that the users are ordered based on their distance from BS such that $\Upsilon_{S}<\Upsilon_{P}$. Therefore, the power allocation for NOMA transmission is performed such that $\alpha_{P}>\alpha_{S}$ to ensure that $U_{P}$ is given the highest priority. We assume in this work that the fading is slow, which implies that the power coefficients can be fixed for a packet interval.

\section{PU Receiver}

Since PU is given the maximum power coefficient, its detector is a conventional maximum likelihood detector (MLD) regard- less of TM because the SU signal is treated as an unknown additive noise. Therefore, assuming that CSI at the receiver (CSIR) is known perfectly, an MLD can be applied to detect the packet in a symbol-by-symbol manner. Thus

$$
\widehat{x}_{P}^{(k)}=\arg \min _{x_{P}^{(k)} \in \chi_{P}}\left|y_{P}^{(k)}-g_{P}^{(k)} x_{P}^{(k)}\right|^{2}
$$

where $x_{P}^{(k)}$ is the trail symbol taken from $\chi_{P}$ codebook of PU. For notational simplicity, the symbol index $k$ will be dropped unless it is necessary to write it explicitly. It is worth noting that the PU receiver can easily identify $\mathrm{TM}_{4}$ by evaluating the feasibility of $\mathrm{TM}_{1}$ using the estimated CSI at the receiver, i.e., check if $P_{B_{P}}^{\mathrm{O}}>\tau_{P}$. If $\mathrm{TM}_{1}$ is infeasible, then $\mathrm{TM}_{4}$ would be the adopted TM.

\section{SU Receiver}

In the absence of side information from the BS about TM, which is the case for uncoordinated networks, the SU has to identify the TM blindly [28]. The TM identification process starts by testing if $P_{B_{S}}^{\mathrm{O}}>\tau_{S}$, if the test result is positive, then the decision will be in favor of $\mathrm{TM}_{4}$. Otherwise, the SU receiver should decide if the received signal corresponds to OMA $\left(\mathrm{TM}_{1}\right.$ or $\left.\mathrm{TM}_{3}\right)$, or NOMA $\left(\mathrm{TM}_{2}\right)$. Therefore, the process can be considered as a binary hypothesis classification [30], which is used to decide about hypotheses and $H_{1}$ [28]. This can be written as

$$
H=\left\{\begin{array}{ll}
H_{0}, & \mathrm{TM}_{1} \text { or } \mathrm{TM}_{3} \\
H_{1}, & \mathrm{TM}_{2}
\end{array} .\right.
$$

The SU receiver can distinguish between $\mathrm{TM}_{1}$ and $\mathrm{TM}_{3}$ by using the upper layers functionalities such as network layer to either keep the packet or drop it based on the destination address, which is assumed to be highly protected. Once the classification problem in (3) is solved, the receiver will user the appropriate detector, i.e. MLD for SU-OMA or SIC for NOMA. Assuming perfect CSIR, the detection process using MLD is similar to that in (2), whereas the detection using SIC can be expressed by

$$
\widehat{x}_{S}=\arg \min _{x_{k} \in \chi_{S}}\left|y_{S}-g_{S} \sqrt{\alpha_{P}} \widehat{x}_{P}-g_{S} \sqrt{\alpha_{S}} x_{k}\right|^{2} .
$$

\section{E. Instantaneous BER Expressions}

The instantaneous BER equations are listed in this subsection. The OMA BER assuming BPSK is given as

$$
P_{B_{j}}^{\mathrm{O}}=Q\left(\sqrt{\gamma_{j}}\right)
$$

while the PU NOMA BER is given by [3]

$$
P_{B_{P}}^{\mathrm{N}}=\frac{1}{2} Q\left(\sqrt{c_{1, P}^{2} \gamma_{P}}\right)+\frac{1}{2} Q\left(\sqrt{c_{2, P}^{2} \gamma_{P}}\right)
$$

where $c_{1, P}=\sqrt{\alpha_{P}}-\sqrt{\alpha_{S}}$, and $c_{2, P}=\sqrt{\alpha_{P}}+\sqrt{\alpha_{S}}$. On the other hand, the SU NOMA BER is [3]

$$
P_{B_{S}}^{\mathrm{N}}=\sum_{r=1}^{5} v_{r} Q\left(\sqrt{c_{r, S}^{2} \gamma_{S}}\right)
$$

where $\mathbf{v}=\frac{1}{2}[2,1,1,-1,-1], c_{1, S}=\sqrt{\alpha_{S}}, c_{2, S}=2 \sqrt{\alpha_{P}}+$ $\sqrt{\alpha_{S}}, c_{3, S}=\sqrt{\alpha_{P}}-\sqrt{\alpha_{S}}, c_{4, S}=\sqrt{\alpha_{P}}+\sqrt{\alpha_{S}}$ and $c_{5, S}=$ $2 \sqrt{\alpha_{P}}-\sqrt{\alpha_{S}}$. 


\section{F. Transmission Modes Probabilities}

To find the TMs probabilities, the SNR threshold $\rho_{j}^{m}$ for each segment of the conditions shown in Table I must be found, and then integrated over the PDF of the instantaneous SNR. Since the instantaneous BER of NOMA is a sum of $Q$-function terms with different arguments, it is difficult to find SNR analytically using the BER formula. Therefore, it is suggested to find the SNR thresholds numerically or graphically.

1) $\mathrm{TM}_{1}:$ The condition in Table I can be written in terms of instantaneous SNR and SNR thresholds as follows,

$$
\left\{\left\{\gamma_{P} \geq \rho_{P}^{\mathrm{O}}\right\} \cap\left\{\gamma_{P}<\rho_{P}^{\mathrm{N}}\right\}\right\} \cup\left\{\left\{\gamma_{P} \geq \rho_{P}^{\mathrm{N}}\right\} \cap\left\{\gamma_{S}<\rho_{S}^{\mathrm{N}}\right\}\right\}
$$

Therefore, the probability of $\mathrm{TM}_{1}$ can be expressed as

$$
\begin{aligned}
& \operatorname{Pr}\left(\mathrm{TM}_{1}\right)=\operatorname{Pr}\left(\rho_{P}^{\mathrm{O}} \leq \gamma_{P}<\rho_{P}^{\mathrm{N}}\right)+\operatorname{Pr}\left(\gamma_{P} \geq \rho_{P}^{\mathrm{N}}, \gamma_{S}<\rho_{S}^{\mathrm{N}}\right) \\
& =F_{\gamma_{P}}\left(\rho_{P}^{\mathrm{N}}\right)-F_{\gamma_{P}}\left(\rho_{P}^{\mathrm{O}}\right) \\
& +\left(1-F_{\gamma_{P}}\left(\rho_{P}^{\mathrm{N}}\right)\right)\left(F_{\gamma_{S}}\left(\rho_{S}^{\mathrm{N}}\right)\right)
\end{aligned}
$$

where $F_{\gamma_{j}}\left(\gamma_{j}\right)=1-\exp \left(-\gamma_{j} / \bar{\gamma}_{j}\right)$.

2) $\mathrm{TM}_{2}$ : The condition can be written in terms of the instantaneous SNR and SNR thresholds,

$$
\left\{\gamma_{P} \geq \rho_{P}^{\mathrm{N}}\right\} \cap\left\{\gamma_{S} \geq \rho_{S}^{\mathrm{N}}\right\}
$$

Therefore, the probability of $\mathrm{TM}_{2}$ can be expressed as

$$
\begin{aligned}
\operatorname{Pr}\left(\mathrm{TM}_{2}\right) & =\operatorname{Pr}\left(\gamma_{P} \geq \rho_{P}^{\mathrm{N}}, \gamma_{S} \geq \rho_{S}^{\mathrm{N}}\right) \\
& =\left(1-F_{\gamma_{P}}\left(\rho_{P}^{\mathrm{N}}\right)\right)\left(1-F_{\gamma_{S}}\left(\rho_{S}^{\mathrm{N}}\right)\right) .
\end{aligned}
$$

3) $\mathrm{TM}_{3}$ : The condition can be written in terms of the instantaneous SNR and SNR thresholds,

$$
\left\{\gamma_{P}<\rho_{P}^{\mathrm{O}}\right\} \cap\left\{\gamma_{S} \geq \rho_{S}^{\mathrm{O}}\right\}
$$

Therefore, the probability of $\mathrm{TM}_{3}$ can be expressed as

$$
\begin{aligned}
\operatorname{Pr}\left(\mathrm{TM}_{3}\right) & =\operatorname{Pr}\left(\gamma_{P}<\rho_{P}^{\mathrm{O}}, \gamma_{S} \geq \rho_{S}^{\mathrm{O}}\right) \\
& =\left(F_{\gamma_{P}}\left(\rho_{P}^{\mathrm{O}}\right)\right)\left(1-F_{\gamma_{S}}\left(\rho_{S}^{\mathrm{O}}\right)\right) .
\end{aligned}
$$

4) $\mathrm{TM}_{4}$ : The condition can be written in terms of the instantaneous SNR and SNR thresholds,

$$
\left\{\gamma_{P}<\rho_{P}^{\mathrm{O}}\right\} \cap\left\{\gamma_{S}<\rho_{S}^{\mathrm{O}}\right\}
$$

Therefore, the probability of $\mathrm{TM}_{3}$ can be expressed as

$$
\operatorname{Pr}\left(\mathrm{TM}_{4}\right)=\operatorname{Pr}\left(\gamma_{P}<\rho_{P}^{\mathrm{O}}, \gamma_{S}<\rho_{S}^{\mathrm{O}}\right)=F_{\gamma_{P}}\left(\rho_{P}^{\mathrm{O}}\right) F_{\gamma_{S}}\left(\rho_{S}^{\mathrm{O}}\right)
$$

\section{Multiple ACCess Classifier}

This section starts with an overview about the decision rules used for the multiple access classifier, which is employed at the SU. Moreover, it defines the performance metrics for the classifier. In addition, it shows the analysis for the BPSK modulation scheme, which is considered for analytical tractability.

\section{A. Overview of Decision Rules}

The classifier tests the binary hypothesis in (3) and makes the decision based on the entire packet observations because the TM is adapted on a packet-by-packet basis. The optimal classifier is the likelihood-ratio test (LRT), which is based on the PDF of the two hypotheses. The PDF of each hypothesis is simply the sum of the possible constellation points' PDFs multiplied by a factor reflecting its weight. Since we assume equally likely symbols within each hypothesis, then the PDF reduces to the average of the constellation points' PDFs which can be described as

$$
f_{\mathbf{y}_{S}}\left(\mathbf{y}_{S} \mid H ; \mathbf{G}_{S}\right)=\frac{1}{\zeta} \prod_{l=1}^{L} \sum_{m=1}^{M_{i}} \exp \left(-\frac{\left|y_{S}^{(l)}-g_{S}^{(l)} A_{m}^{(i)}\right|^{2}}{2 \sigma_{w}^{2}}\right)
$$

where $\zeta=\left(2 \pi \sigma_{w}^{2} M_{i}\right)^{L}, M_{i}$ is the modulation order or the number of constellation points for a given multiple access technique, $y^{(l)}$ is the $l$ th observation in the packet and $A_{m}^{(i)}$ is the $m$ th constellation point's amplitude. Here, the product exists because the observations are independent from each other given that the channel gain faced by all observations is the same, however, independent noise samples are faced. The LRT is the ratio of likelihoods compared to a threshold related to the prior probabilities of the two hypotheses, i.e.

$$
\Lambda_{\mathrm{LRT}}=\frac{f_{\mathbf{y}_{S}}\left(\mathbf{y}_{S} \mid H_{1} ; \mathbf{G}_{S}\right)}{f_{\mathbf{y}_{S}}\left(\mathbf{y}_{S} \mid H_{0} ; \mathbf{G}_{S}\right)} \underset{H_{0}}{\gtrless} \frac{H_{1}}{\operatorname{Pr}\left(H_{0}\right)} \triangleq \frac{\operatorname{Pr}\left(\mathrm{TM}_{3}\right)}{\operatorname{Pr}\left(\mathrm{TM}_{2}\right)} .
$$

The simplification of (17) results in a complex decision rule. Therefore, an alternative suboptimal rule is proposed, which is the ML estimation for each observation. The rule is suboptimal because $\operatorname{Pr}\left(H_{0}\right) \neq \operatorname{Pr}\left(H_{1}\right)$, whereas the maximum a posterior probability (MAP) rule would give an optimal solution based on each observation. The ML rule finds the most likely multiple access by searching through all possible constellation points and selecting the point with the minimum Euclidean distance. The estimated hypothesis for the $l$ th observation can be written as

$$
\begin{aligned}
\widehat{H}^{(l)} & =\arg \max _{m \in\left(1, M_{i}\right)} \frac{1}{2 \pi \sigma_{w}^{2}} \exp \left(-\frac{\left|y_{S}^{(l)}-g_{S}^{(l)} A_{m}^{(k)}\right|^{2}}{2 \sigma_{w}^{2}}\right) \\
& =\arg \min _{x_{i} \in\left(\chi_{0}, \chi_{1}\right)}\left|y_{S}^{(l)}-g_{S}^{(l)} x_{i}\right|^{2}
\end{aligned}
$$

where $\chi_{0}$ is a set of both users' codebooks such that $\chi_{0} \in$ $\left(\chi_{P}, \chi_{S}\right)$, while $\chi_{1}$ is the NOMA codebook resulting from the $\mathrm{SC}$ of the two users codebooks. The decision on these local observations should be combined to have a global decision. Consequently, hard decision counting rules are proposed and will be discussed in the next section.

\section{B. Performance Metrics for Classifier}

The classifier is imperfect, therefore, erroneous decisions which degrade the performance might take place and degrade the performance. These errors are:

- Miss-detection: $P_{\mathrm{m}}^{(l)}=\operatorname{Pr}\left(\widehat{H}^{(l)}=H_{0} \mid H_{1}, \mathrm{TM}_{2}\right)$.

- False alarm 1: $P_{\mathrm{fa}_{1}}^{(l)}=\operatorname{Pr}\left(\hat{H}^{(l)}=H_{1} \mid H_{0}, \mathrm{TM}_{1}\right)$.

- False alarm 2: $P_{\mathrm{fa}_{2}}^{(l)}=\operatorname{Pr}\left(\widehat{H}^{(l)}=H_{1} \mid H_{0}, \mathrm{TM}_{3}\right)$.

Note that all the previous metrics represent the decision on a single observation, which is known as the local decision and denoted with a small letter subscript. However, after combining 
the observations, the performance metric is denoted with a capital letter subscript. The false alarm 1 event can be ignored as the destination address field will be checked. Nonetheless, the miss-detection and false alarm 2 (false alarm for short) events must be considered as both affect the throughput directly. The former indicates that the packet will be lost because SIC is not used, while the latter indicates that SIC will be used for decoding which will reduce the signal strength leading to a performance degradation.

\section{Classifier Analysis}

The classifier is based on the ML rule which divides the signal space into two regions representing NOMA and OMA. This can be seen in Fig. 2 where BPSK is used for OMA while NOMA is made of two users with BPSK. It is worth mentioning that selecting higher order modulation would make the regions as polygons resulting in intractable analysis. Further,

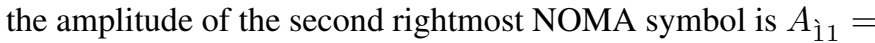
$-\sqrt{\alpha_{S}}+\sqrt{\alpha_{P}}$. Here, the conditional miss-detection and false alarm probabilities will be derived. For notational simplicity, the index of the observation will be dropped, i.e. $P_{\mathrm{fa}_{2}}, P_{\mathrm{m}}$.

1) $P_{\mathrm{fa}_{2}} \mid \gamma_{S}$ Analysis: The event of false alarm will take place if OMA was transmitted but the classifier estimates it as a NOMA signal. Two cases are there but only one case will be studied because of symmetry.

Case 1: Amplitude $B$ is transmitted by the BS. Therefore, the probability that the signal falls in the NOMA region, given that $B$ is transmitted, represents the probability of false alarm, which is

$$
\begin{aligned}
& P_{\mathrm{fa}_{2}}^{(1)} \mid \gamma_{S}=\operatorname{Pr}\left(\tilde{w}>\frac{A_{11}-B}{2}\right)+\operatorname{Pr}\left(\tilde{w}<-\frac{B-A_{\grave{1} 1}}{2}\right) \\
& -\operatorname{Pr}\left(-\frac{3 B+A_{11}}{2}<\tilde{w}<-\frac{3 B+A_{\grave{1} 1}}{2}\right)
\end{aligned}
$$

where $\tilde{w} \triangleq \Re[\tilde{w}]$. Considering the first term only to show how the integration over the AWGN PDF is done, then

$$
\begin{aligned}
\operatorname{Pr}\left(\tilde{w}>\frac{A_{11}-B}{2} \gamma_{S}\right) & =\frac{1}{\sqrt{2 \pi \sigma_{\tilde{w}}^{2}}} \int_{\frac{A_{11}-B}{2} \gamma_{S}}^{\infty} \exp \left(\frac{-\tilde{w}^{2}}{2}\right) \mathrm{d} \tilde{w} \\
& =Q\left(\sqrt{c_{1, C}^{2} \gamma_{S}}\right)
\end{aligned}
$$

where $\sigma_{\tilde{w}}^{2}=0.5 N_{0}, Q(\cdot)$ is the Gaussian function and $c_{1, C}=$ $\frac{A_{11}-B}{2}$. Therefore, simplifying (19) results in

$$
\begin{aligned}
P_{\mathrm{fa}_{2}}^{(1)} \mid \gamma_{S} & =Q\left(\sqrt{c_{1, C}^{2} \gamma_{S}}\right)+Q\left(\sqrt{c_{2, C}^{2} \gamma_{S}}\right) \\
& -Q\left(\sqrt{c_{3, C}^{2} \gamma_{S}}\right)+Q\left(\sqrt{c_{4, C}^{2} \gamma_{S}}\right)
\end{aligned}
$$

where $c_{2, C}=\frac{B-A_{\text {ì }}}{2}, c_{3, C}=\frac{3 B+A_{\text {\1 }}}{2}$ and $c_{4, C}=\frac{3 B+A_{11}}{2}$. As mentioned previously, the two cases are symmetric. Hence, $P_{\mathrm{fa}_{2}} \mid \gamma_{S}$ is identical to (21).

2) $P_{\mathrm{m}} \mid \gamma_{S}$ Analysis: The event of miss-detection will take place if NOMA was transmitted but the classifier estimates it as OMA signal. Therefore, four cases are there but only two cases are studied because of symmetry.

Case 1: Amplitude $A_{11}$ is transmitted by the BS. Therefore, the probability that the signal falls in the OMA region, given that

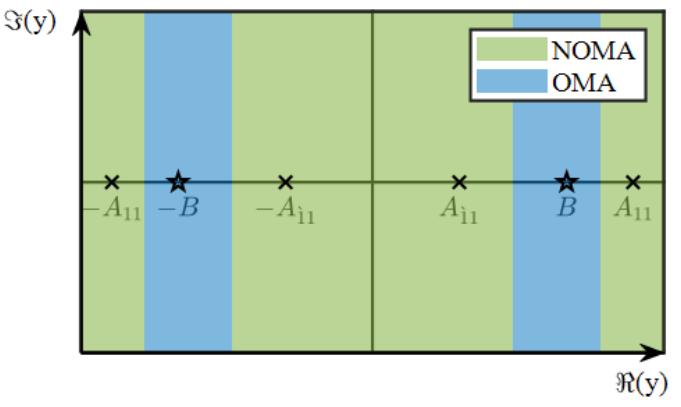

Fig. 2: Signal space diagram for NOMA and OMA.

$A_{11}$ is transmitted, represents the probability of miss-detection for this case which is

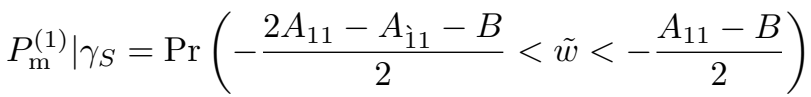

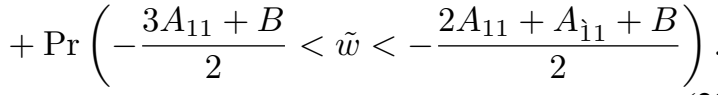

Simplifying (22) results in a sum $Q(\cdot)$ functions that can be written as

$$
\begin{aligned}
P_{\mathrm{m}}^{(1)} \mid \gamma_{S} & =Q\left(\sqrt{c_{1, C}^{2} \gamma_{S}}\right)-Q\left(\sqrt{c_{5, C}^{2} \gamma_{S}}\right) \\
& +Q\left(\sqrt{c_{6, C}^{2} \gamma_{S}}\right)-Q\left(\sqrt{c_{7, C}^{2} \gamma_{S}}\right)
\end{aligned}
$$

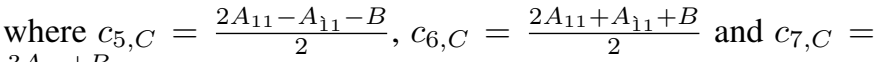
$\frac{3 A_{11}+B}{2}$.

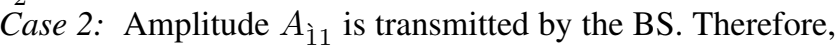
the probability of miss-detection for this case is

$$
\begin{aligned}
& P_{\mathrm{m}}^{(2)} \mid \gamma_{S}=\operatorname{Pr}\left(\frac{B-A_{\grave{1} 1}}{2}<\tilde{w}<\frac{A_{11}+B-2 A_{\grave{1} 1}}{2}\right)
\end{aligned}
$$

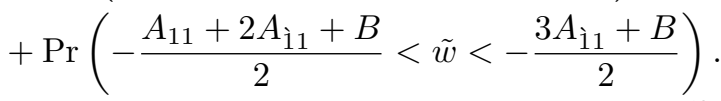

Simplifying (24) results in a sum $Q(\cdot)$ functions that can be written as

$$
\begin{aligned}
P_{\mathrm{m}}^{(2)} \mid \gamma_{S} & =Q\left(\sqrt{c_{2, C}^{2} \gamma_{S}}\right)-Q\left(\sqrt{c_{8, C}^{2} \gamma_{S}}\right) \\
& +Q\left(\sqrt{c_{9, C}^{2} \gamma_{S}}\right)-Q\left(\sqrt{c_{10, C}^{2} \gamma_{S}}\right)
\end{aligned}
$$

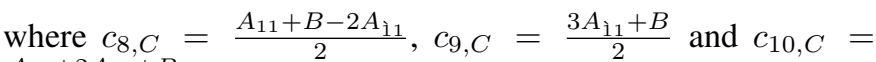
$\frac{A_{11}+2 A_{11}+B}{2}$. For brevity, Case 3 and Case 4 analyses are omitted because they are identical to Case 2 and Case 1, respectively. Therefore, the conditional probability of miss-detection can be written as

$$
P_{\mathrm{m}}\left|\gamma_{S}=\frac{1}{2} \sum_{r=1}^{2} P_{\mathrm{m}}^{(r)}\right| \gamma_{S}=\sum_{r=1}^{10} v_{r} Q\left(\sqrt{c_{r, C}^{2} \gamma_{S}}\right)
$$

where $\mathbf{v}=(1 / 2)[1,1,0,0,-1,1,-1,-1,1,-1]$.

3) Unconditional Analysis: Removing the conditioning requires integration over the PDF of $\gamma_{S}$ and this is relatively simple for the local observations as the $P_{\mathrm{m}} \mid \gamma_{S}$ or $P_{\mathrm{fa}_{2}} \mid \gamma_{S}$ are sum of $Q(\cdot)$ terms [3, Eq. (46)]. Therefore, the unconditional 
local probability of miss-detection or false alarm can be found by considering the following integral,

$$
I_{1}=\frac{1}{1-F_{\gamma_{S}}(\rho)} \int_{\rho}^{\infty} Q\left(\sqrt{c^{2} \gamma_{S}}\right) f_{\gamma_{S}}\left(\gamma_{S}\right) \mathrm{d} \gamma_{S}
$$

where $f_{\gamma_{j}}\left(\gamma_{j}\right)=\exp \left(\gamma_{j} / \bar{\gamma}_{j}\right) / \bar{\gamma}_{j}$, and the integration's lower limit is $\rho$ rather than 0 because there is a condition on $\gamma_{S}$ that is related to either $\mathrm{TM}_{2}$ or $\mathrm{TM}_{3}$ as can be seen in (11) and (13). In addition, the subscript is dropped from $\rho$ to be generic for both metrics. This factor depends on the SNR threshold defined in the previous section where $\rho=\rho_{S}^{\mathrm{N}}$ for $P_{\mathrm{m}}$, while $\rho=\rho_{S}^{\mathrm{O}}$ for $P_{\mathrm{fa}_{2}}$. The integral in (27) can be re-written as

$$
\begin{gathered}
I_{1}=\frac{1}{1-F_{\gamma_{S}}(\rho)} \int_{0}^{\infty} Q\left(\sqrt{c^{2} \gamma_{S}}\right) f_{\gamma_{S}}\left(\gamma_{S}\right) \mathrm{d} \gamma_{S} \\
\quad-\frac{1}{1-F_{\gamma_{S}}(\rho)} \int_{0}^{\rho} Q\left(\sqrt{c^{2} \gamma_{S}}\right) f_{\gamma_{S}}\left(\gamma_{S}\right) \mathrm{d} \gamma_{S} .
\end{gathered}
$$

Evaluating the two integrals gives,

$$
\begin{aligned}
I_{1} & =\frac{1-\sqrt{\xi^{-1}}}{2\left(1-F_{\gamma_{S}}(\rho)\right)}-\frac{1}{1-F_{\gamma_{S}}(\rho)} \\
& \times\left[\frac{1}{2}-Q\left(\sqrt{c^{2} \rho}\right) \exp \left(\frac{-\rho}{\bar{\gamma}_{S}}\right)+\frac{2 Q\left(\sqrt{\rho c^{2} \xi}\right)-1}{2 \sqrt{\xi}}\right]
\end{aligned}
$$

where $\xi=\frac{c^{2} \bar{\gamma}_{S}+2}{c^{2} \bar{\gamma}_{S}}$. Therefore, $P_{\mathrm{fa}_{2}}$ can be written as

$$
\begin{aligned}
& P_{\mathrm{fa}_{2}}=\frac{1}{\left(1-F_{\gamma_{S}}\left(\rho_{S}^{\mathrm{O}}\right)\right)} \sum_{r=1}^{4} v_{r} \times\left[\frac{1-\sqrt{\xi_{r}^{-1}}}{2}-\frac{1}{2}+\right. \\
& \left.Q\left(\sqrt{c_{r, C}^{2} \rho_{S}^{\mathrm{O}}}\right) \exp \left(\frac{-\rho_{S}^{\mathrm{O}}}{\bar{\gamma}_{S}}\right)-\frac{2 Q\left(\sqrt{c_{r, C}^{2} \rho_{S}^{\mathrm{O}} \xi_{r}}\right)-1}{2 \sqrt{\xi_{r}}}\right]
\end{aligned}
$$

where $\xi_{r}=\frac{c_{r, C}^{2} \bar{\gamma}_{S}+2}{c_{r, C}^{2} \bar{\gamma}_{S}}$ and $\mathbf{v}=[1,1,-1,1]$. Furthermore, $P_{\mathrm{m}}$ can be written as

$$
\begin{aligned}
& P_{\mathrm{m}}=\frac{1}{\left(1-F_{\gamma_{S}}\left(\rho_{S}^{\mathrm{N}}\right)\right)} \sum_{r=1}^{10} v_{r} \times\left[\frac{1-\sqrt{\xi_{r}^{-1}}}{2}-\frac{1}{2}+\right. \\
& \left.Q\left(\sqrt{c_{r, C}^{2} \rho_{S}^{\mathrm{N}}}\right) \exp \left(\frac{-\rho_{S}^{\mathrm{N}}}{\bar{\gamma}_{S}}\right)-\frac{2 Q\left(\sqrt{c_{r, C}^{2} \rho_{S}^{\mathrm{N}} \xi_{r}}\right)-1}{2 \sqrt{\xi_{r}}}\right]
\end{aligned}
$$

where $\mathbf{v}=(1 / 2)[1,1,0,0,-1,1,-1,-1,1,-1]$ On the other hand, the case is not simple for the global miss-detection probability, $P_{\mathrm{M}} \mid \gamma_{S}$, and global false alarm probability, $P_{\mathrm{FA}_{2}} \mid \gamma_{S}$, as the integration will involve higher power orders as will be seen in the following sections.

\section{Counting Rules Analysis}

The counting rules are considered hard decision rules. Several counting rules are studied in this section and they are: AND, OR, Majority and $K$-out- $L$ rules. This section will start by stating the decision-rule statistics of each counting rule then it will derive the conditional and unconditional global probabilities of missdetection and global false alarm.

\section{A. Decision-Rule Statistics}

Let us start by defining the indicator function to denote $\widehat{H}^{(l)}$ numerically, this can be expressed as follows,

$$
\mathbf{1}_{H_{1}}^{(l)}\left(\widehat{H}^{(l)}\right)=\left\{\begin{array}{ll}
1 & \text { if } \widehat{H}^{(l)}=H_{1} \\
0 & \text { if } \widehat{H}^{(l)} \neq H_{1}
\end{array} .\right.
$$

In addition, the total number of observations decided as $H_{1}$ is $O=\sum_{l=1}^{L} \mathbf{1}_{H_{1}}^{(l)}$. Therefore, the decision-rule statistics can be written for each counting rule as follows:

1) AND Rule:

$$
\widehat{H}= \begin{cases}H_{0} & \text { if } 0 \leq O<L \\ H_{1} & \text { if } O=L\end{cases}
$$

which implies that all local observations must be NOMA to have a global NOMA observation, otherwise it is a global OMA observation.

2) OR Rule:

$$
\widehat{H}= \begin{cases}H_{0} & \text { if } O=0 \\ H_{1} & \text { if } O \geq 1\end{cases}
$$

which indicates that at least one local observation must be NOMA to have a global decision of NOMA. otherwise it is a global OMA observation.

3) K-out-of-L Rule:

$$
\widehat{H}= \begin{cases}H_{0} & \text { if } O<\lceil K L\rceil \\ H_{1} & \text { if } O \geq\lceil K L\rceil\end{cases}
$$

which indicates that at least $\lceil K L\rceil$ local observations must be NOMA to have a global NOMA observation, otherwise it is a global OMA observation. Note that the Majority rule is a special case of the $K$-out-of- $L$ rule when $K=1 / 2$.

\section{B. Conditional Performance Analysis}

The following derivations employ the fact that the observations are conditionally independent from each other as the noise samples are independent. In general, the conditional global probability of false alarm is given as $P_{\mathrm{FA}_{2}} \mid \gamma_{S}=$ $\operatorname{Pr}\left(\widehat{H}=H_{1} \mid H_{0}, \mathrm{TM}_{3}\right)=1-\operatorname{Pr}\left(\widehat{H}=H_{0} \mid H_{0}, \mathrm{TM}_{3}\right)$, while the conditional global probability of miss-detection is given as $P_{\mathrm{M}} \mid \gamma_{S}=\operatorname{Pr}\left(\widehat{H}=H_{0} \mid H_{1}\right)=1-$ $\operatorname{Pr}\left(\widehat{H}=H_{1} \mid H_{1}\right)$. 
1) AND Rule: The whole packet is in false alarm when all local observations are in false alarm. Therefore,

$$
P_{\mathrm{FA}_{2}}\left|\gamma_{S}=\prod_{l=1}^{L} P_{\mathrm{fa}_{2}}^{(l)}\right| \gamma_{S}=\left(P_{\mathrm{fa}_{2}} \mid \gamma_{S}\right)^{L}
$$

On the other hand, $P_{\mathrm{M}} \mid \gamma_{S}$ is

$$
P_{\mathrm{M}} \mid \gamma_{S}=1-\prod_{l=1}^{L}\left(1-P_{\mathrm{m}}^{(l)} \mid \gamma_{S}\right)=1-\left(1-P_{\mathrm{m}} \mid \gamma_{S}\right)^{L}
$$

which can be expanded using the Binomial theorem which states that $(a+b)^{n}=\sum_{k=0}^{n}\left(\begin{array}{l}n \\ k\end{array}\right) a^{n-k} b^{k}$. Therefore,

$$
P_{\mathrm{M}} \mid \gamma_{S}=1-\sum_{r=0}^{L}\left(\begin{array}{l}
L \\
r
\end{array}\right)(-1)^{r}\left(P_{\mathrm{m}} \mid \gamma_{S}\right)^{r} .
$$

2) OR Rule: The whole packet is in false alarm when at least one local observation is in false alarm. Therefore, $P_{\mathrm{FA}_{2}} \mid \gamma_{S}$ can be expressed by

$$
P_{\mathrm{FA}_{2}} \mid \gamma_{S}=1-\prod_{l=1}^{L}\left(1-P_{\mathrm{fa}_{2}}^{(l)} \mid \gamma_{S}\right)=1-\left(1-P_{\mathrm{fa}_{2}} \mid \gamma_{S}\right)^{L} .
$$

which can be written as

$$
P_{\mathrm{FA}_{2}} \mid \gamma_{S}=1-\sum_{r=0}^{L}\left(\begin{array}{c}
L \\
r
\end{array}\right)(-1)^{r}\left(P_{\mathrm{fa}_{2}} \mid \gamma_{S}\right)^{r} .
$$

On the other hand, the whole packet will be in a miss-detection event if all observations are in miss-detection. As a result, $P_{\mathrm{M}} \mid \gamma_{S}$ is expressed as

$$
P_{\mathrm{M}}\left|\gamma_{S}=\prod_{l=1}^{L} P_{\mathrm{m}}^{(l)}\right| \gamma_{S}=\left(P_{\mathrm{m}} \mid \gamma_{S}\right)^{L}
$$

3) K-out-of-L Rule: The whole packet is in false alarm when at least $\lceil K L\rceil$ local observations are in false alarm. Therefore, $P_{\mathrm{FA}_{2}} \mid \gamma_{S}$ can be expressed by

$$
\begin{aligned}
P_{\mathrm{FA}_{2}} \mid \gamma_{S} & =\sum_{l=\lceil K L\rceil}^{L}\left(\begin{array}{c}
L \\
l
\end{array}\right)\left(P_{\mathrm{fa}_{2}} \mid \gamma_{S}\right)^{l}\left(1-P_{\mathrm{fa}_{2}} \mid \gamma_{S}\right)^{L-l} \\
& =\sum_{l=\lceil K L\rceil}^{L} \sum_{r=0}^{L-l}\left(\begin{array}{c}
L \\
l
\end{array}\right)\left(\begin{array}{c}
L-l \\
r
\end{array}\right)(-1)^{r}\left(P_{\mathrm{fa}_{2}} \mid \gamma_{S}\right)^{l+r} .
\end{aligned}
$$

Moreover, the whole packet will be in miss-detection if at most $\lceil K L\rceil$ local observations are in miss-detection. Therefore, $P_{\mathrm{M}} \mid \gamma_{S}$ is

$$
\begin{aligned}
P_{\mathrm{M}} \mid \gamma_{S} & =1-\sum_{l=\lceil K L\rceil}^{L}\left(\begin{array}{c}
L \\
l
\end{array}\right)\left(1-P_{\mathrm{m}}\right)^{l}\left(P_{\mathrm{m}}\right)^{L-l} \\
& =1-\sum_{l=\lceil K L\rceil}^{L} \sum_{r=0}^{l}\left(\begin{array}{l}
L \\
l
\end{array}\right)\left(\begin{array}{l}
l \\
r
\end{array}\right)(-1)^{r}\left(P_{\mathrm{m}}\right)^{L+r-l} .
\end{aligned}
$$

\section{Unconditional Performance Analysis}

Removing the conditions from previous global performance metrics require the integration over the PDF of the channel such that

$$
\begin{aligned}
P_{\mathrm{FA}_{2}} & =\frac{1}{1-F_{\gamma_{S}}\left(\rho_{S}^{\mathrm{O}}\right)} \int_{\rho_{S}^{\mathrm{O}}}^{\infty} P_{\mathrm{FA}_{2}} \mid \gamma_{S} f_{\gamma_{S}}\left(\gamma_{S}\right) \mathrm{d} \gamma_{S} \\
P_{\mathrm{M}} & =\frac{1}{1-F_{\gamma_{S}}\left(\rho_{S}^{\mathrm{N}}\right)} \int_{\rho_{S}^{\mathrm{N}}}^{\infty} P_{\mathrm{M}} \mid \gamma_{S} f_{\gamma_{S}}\left(\gamma_{S}\right) \mathrm{d} \gamma_{S} .
\end{aligned}
$$

It is quite challenging to solve these integrals because the local probabilities of miss-detection and false alarm are in a form of sum of $Q(\cdot)$ terms. Moreover, the conditional counting rules expressions, i.e. $P_{\mathrm{FA}_{2}} \mid \gamma_{S}$ and $P_{\mathrm{M}} \mid \gamma_{S}$, being a sum of $Q$ terms make the analysis intractable. Therefore, it is suggested to approximate $Q$ function, as well as the local probabilities of miss-detection and false alarm using the most dominant terms.

In general, the conditional global probabilities of false alarm and miss-detection have one of two forms which are

$$
\begin{gathered}
\mathcal{P}_{1} \mid \gamma_{S}=1-\left(1-P \mid \gamma_{S}\right)^{L} \\
\mathcal{P}_{2} \mid \gamma_{S}=\left(P \mid \gamma_{S}\right)^{L}
\end{gathered}
$$

where $\mathcal{P}_{1} \mid \gamma_{S}$ includes $P_{\mathrm{FA}_{2}} \mid \gamma_{S}$ for OR rule and $P_{\mathrm{M}} \mid \gamma_{S}$ for AND rule, whereas $\mathcal{P}_{2} \mid \gamma_{S}$ includes $P_{\mathrm{FA}_{2}} \mid \gamma_{S}$ for OR and $K$-out-of- $L$ rules as well as $P_{\mathrm{M}} \mid \gamma_{S}$ for AND and $K$-out-of- $L$ rules.

1) Analysis of Form 1: Considering (46), it is noted that the most dominant term in $P_{\mathrm{fa}_{2}} \mid \gamma_{S}$ and $P_{\mathrm{m}} \mid \gamma_{S}$ is sufficient to provide accurate results. Therefore, by observing (21), it is noted that the dominant term is related to the argument of the $Q$ function with the minimum value. Hence, $c_{\min , C}=c_{1, C}$. Therefore, the local false alarm probability can be approximated as

$$
P_{\mathrm{fa}_{2}} \mid \gamma_{S} \approx Q\left(\sqrt{c_{1, C}^{2} \gamma_{S}}\right)
$$

Similarly, by observing (26), the expression can be approximated as

$$
P_{\mathrm{m}} \mid \gamma_{S} \approx \frac{1}{2} Q\left(\sqrt{c_{1, C}^{2} \gamma_{S}}\right)
$$

Considering the previous approximation, the conditional global classification errors have a similar form which is related to power of $Q(\cdot)$ function, i.e. $Q^{r}(\cdot)$. Therefore, to remove the condition and get the average global classification error, an integration over $f_{\gamma_{S}}\left(\gamma_{S}\right)$ should take place, and this can be written as

$$
\begin{aligned}
\mathcal{P}_{1}=1-\frac{1}{1-F_{\gamma_{S}}(\rho)} \sum_{r=0}^{L}\left(\begin{array}{l}
L \\
r
\end{array}\right)(-\eta)^{r} \\
\times \int_{\rho}^{\infty} Q^{r}\left(\sqrt{c^{2} \gamma_{S}}\right) f_{\gamma_{S}}\left(\gamma_{S}\right) \mathrm{d} \gamma_{S}
\end{aligned}
$$

where $c=c_{1, C}, \eta=1 / 2$ for miss-detection, while $\eta=1$ for false alarm. Since $Q$ is raised to a power, solving (50) for arbitrary an power is still an open research question. Therefore, an approximation of $Q$ is suggested instead, which is

$$
Q(z) \approx \frac{\exp \left(-\frac{z^{2}}{2}\right)}{\sqrt{2 \pi\left(z^{2}+1\right)}} .
$$


By using the approximation shown in (51), the integral shown in (50) can be re-written as follows,

$$
I_{2}=\frac{1}{\bar{\gamma}_{S}} \int_{\rho}^{\infty}\left(\frac{\exp \left(-\frac{c^{2} \gamma_{S}}{2}\right)}{\sqrt{2 \pi\left(c^{2} \gamma_{S}+1\right)}}\right)^{r} \exp \left(-\frac{\gamma_{S}}{\bar{\gamma}_{S}}\right) \mathrm{d} \gamma_{S} .
$$

By letting $t=\sqrt{c^{2} \gamma_{S}+1}, \gamma_{S}=\frac{t^{2}-1}{c^{2}}$, this can be written as

$$
\begin{aligned}
I_{2}= & \frac{2 \exp \left(\frac{r}{2}+\frac{1}{c^{2} \bar{\gamma}_{S}}\right)}{c^{2} \bar{\gamma}_{S} \sqrt{(2 \pi)^{r}}} \\
& \times \int_{\sqrt{c^{2} \rho+1}}^{\infty} \frac{1}{t^{r-1}} \exp \left(-t^{2}\left[\frac{r}{2}+\frac{1}{c^{2} \bar{\gamma}_{S}}\right]\right) \mathrm{d} t
\end{aligned}
$$

where the integral shown in (53) has a closed-form according to [34, p. 369]. Therefore, the expression can be written as

$$
I_{2}=\frac{\exp \left(\frac{r}{2}+\frac{1}{c^{2} \bar{\gamma}_{S}}\right) \Gamma\left(\frac{2-r}{2},\left[\frac{r}{2}+\frac{1}{c^{2} \bar{\gamma}_{S}}\right]\left(c^{2} \rho+1\right)\right)}{c^{2} \bar{\gamma}_{S} \sqrt{(2 \pi)^{r}}\left(\frac{r}{2}+\frac{1}{c^{2} \bar{\gamma}_{S}}\right)^{\frac{2-r}{2}}} .
$$

Finally, $\mathcal{P}_{1}$ can be found by replacing the integration in (50) by (54). Thus,

$$
\mathcal{P}_{1}=1-\frac{1}{1-F_{\gamma_{S}}(\rho)} \sum_{r=0}^{L}\left(\begin{array}{l}
L \\
r
\end{array}\right)(-\eta)^{r} \times I_{2} .
$$

The value of $\rho$ distinguishes between false alarm and missdetection probabilities for OR and AND rules, respectively.

2) Analysis of Form 2: Considering (47), it is noted that the most two dominant terms in $P_{\mathrm{fa}_{2}} \mid \gamma_{S}$ and $P_{\mathrm{m}} \mid \gamma_{S}$ are sufficient to provide accurate results. Therefore, the local false alarm probability can be approximated as

$$
P_{\mathrm{fa}_{2}} \mid \gamma_{S} \approx \sum_{q=1}^{2} Q\left(\sqrt{c_{q, C}^{2} \gamma_{S}}\right) .
$$

Similarly, by observing (26), the expression can be approximated as

$$
P_{\mathrm{m}} \mid \gamma_{S} \approx \frac{1}{2} \sum_{q=1}^{2} Q\left(\sqrt{c_{q, C}^{2} \gamma_{S}}\right) .
$$

This would require using Multinomial theorem to expand the conditional counting rules expressions. Therefore, it is suggested to use the weighted sums of exponential approximations for the $Q$ function to have a closed-form solution. The weights are optimized to minimize the error and can be found in [35]. The approximation of the Gaussian function is given as

$$
Q(z) \approx \sum_{n} a_{n} \exp \left(-b_{n} z^{2}\right)
$$

Therefore, (47) can be written as

$$
\begin{aligned}
\mathcal{P}_{2} \mid \gamma_{S}= & \sum_{N=L}\left(\begin{array}{c}
L \\
N_{1}, \cdots, N_{2 n}
\end{array}\right) \\
& \times \prod_{q=1}^{n} \prod_{t=2 q-1}^{2 q}\left(\eta a_{q}\right)^{N_{t}} \exp \left(-b_{q} N_{t} c_{\zeta, C}^{2} \gamma_{S}\right)
\end{aligned}
$$

where $N=N_{1}+N_{2}+\cdots+N_{2 n}, \zeta=1$ iff $q \leq n$, otherwise $\zeta=2$. This can be written as,

$$
\mathcal{P}_{2} \mid \gamma_{S}=\sum_{N=L}\left(\begin{array}{c}
L \\
N_{1}, \cdots, N_{2 n}
\end{array}\right) \Psi \exp \left(-\gamma_{S} \Delta\right)
$$

where $\Psi=\prod_{q=1}^{n} \prod_{t=2 q-1}^{2 q}\left(\eta a_{q}\right)^{N_{t}}$ and $\Delta=$ $\sum_{q=1}^{n} \sum_{t=2 q-1}^{2 q} b_{q} N_{t} c_{\zeta, C}^{2}$. The unconditional expression can be found by

$$
\begin{aligned}
\mathcal{P}_{2}=\frac{1}{1-F_{\gamma_{S}}(\rho)} \sum_{N=L}\left(\begin{array}{c}
L \\
N_{1}, \cdots, N_{2 n}
\end{array}\right) \\
\quad \times \frac{\Psi}{\bar{\gamma}_{S}} \int_{\rho}^{\infty} \exp \left(-\gamma_{S}\left(\Delta+\frac{1}{\bar{\gamma}_{S}}\right)\right) \mathrm{d} \gamma_{S} .
\end{aligned}
$$

Therefore, the closed-form solution is given as

$$
\begin{aligned}
\mathcal{P}_{2}=\frac{1}{1-F_{\gamma_{S}}(\rho)} & \sum_{N=L}\left(\begin{array}{c}
L \\
N_{1} \cdots, N_{2 n}
\end{array}\right) \\
& \times \frac{\Psi}{\Delta \bar{\gamma}_{S}+1} \exp \left(-\rho\left(\Delta+\frac{1}{\bar{\gamma}_{S}}\right)\right) .
\end{aligned}
$$

It is worth noting that the value of $\rho$ distinguishes between false alarm and miss-detection probabilities for the AND and OR rules, respectively. Furthermore, the same expression can be used for the $K$-out-of- $L$ rule but it needs to account for the summations and constants in (42) and (43). Therefore, $P_{\mathrm{FA}_{2}}$ for the $K$-out-of- $L$ rule can be written as

$$
\begin{aligned}
P_{\mathrm{FA}_{2}}=\frac{1}{1-F_{\gamma_{S}}\left(\rho_{S}^{\mathrm{O}}\right)} \sum_{l=\lceil K L\rceil}^{L} \sum_{r=0}^{L-l}\left(\begin{array}{c}
L \\
l
\end{array}\right)\left(\begin{array}{c}
L-l \\
r
\end{array}\right)(-1)^{r} \\
\times \sum_{N=l+r}\left(\begin{array}{c}
l+r \\
N_{1}, \cdots, N_{2 n}
\end{array}\right) \\
\times \frac{\Psi}{\Delta \bar{\gamma}_{S}+1} \exp \left(-\rho_{S}^{\mathrm{O}}\left(\Delta+\frac{1}{\bar{\gamma}_{S}}\right)\right) .
\end{aligned}
$$

Similarly, $P_{\mathrm{M}}$ for the $K$-out-of- $L$ rule can be written as

$$
\begin{aligned}
& P_{\mathrm{M}}=1-\frac{1}{1-F_{\gamma_{S}}\left(\rho_{S}^{\mathrm{N}}\right)} \sum_{l=\lceil K L\rceil}^{L} \sum_{r=0}^{l}\left(\begin{array}{l}
L \\
l
\end{array}\right)\left(\begin{array}{l}
l \\
r
\end{array}\right)(-1)^{r} \\
& \times \sum_{N=L+r-l}\left(\begin{array}{c}
L+r-l \\
N_{1}, \cdots, N_{2 n}
\end{array}\right) \\
& \times \frac{\Psi}{\Delta \bar{\gamma}_{S}+1} \exp \left(-\rho_{S}^{\mathrm{N}}\left(\Delta+\frac{1}{\bar{\gamma}_{S}}\right)\right) \text {. }
\end{aligned}
$$

\section{Packet Error Rate Analysis}

In this section, the PER will be derived as to quantify the detectors performance at each user. The conditional PER in a block fading channel can be found as follows,

$$
P_{e_{j}}^{m} \mid \gamma_{j}=1-\left(1-P_{B_{j}}^{m}\right)^{L}=1-\sum_{r=0}^{L}\left(\begin{array}{l}
L \\
r
\end{array}\right)(-1)^{r}\left(P_{B_{j}}^{m}\right)^{r} .
$$

This expression have a form identical to Form 1 in (46). 


\section{A. OMA PER}

The PER of PU-OMA case consists of two cases as there are two conditions that either of them lead to PU-OMA transmission. Therefore, the PER can be found as $P_{e_{P}}^{\mathrm{O}}=\operatorname{Pr}($ Case 1$) \times$ $P_{e_{P}}^{\mathrm{O}, \text { Case } 1}+\operatorname{Pr}($ Case 2$) \times P_{e_{P}}^{\mathrm{O}, \text { Case } 2}$. Case 1 is denoted for $\rho_{P}^{\mathrm{O}} \leq \gamma_{P}<\rho_{P}^{\mathrm{N}}$, whereas Case 2 is denoted for $\gamma_{P} \geq$ $\rho_{P}^{\mathrm{N}} \cap \gamma_{S}<\rho_{S}^{\mathrm{N}}$. Hence, $\operatorname{Pr}($ Case 1$)=F_{\gamma_{P}}\left(\rho_{P}^{\mathrm{N}}\right)-F_{\gamma_{P}}\left(\rho_{P}^{\mathrm{O}}\right)$, while $\operatorname{Pr}($ Case 2$)=\left(1-F_{\gamma_{P}}\left(\rho_{P}^{N}\right)\right) F_{\gamma_{S}}\left(\rho_{S}^{N}\right)$. Note that the probabilities of these two events should be normalized by the space probability which is $\operatorname{Pr}($ Case 1$)+\operatorname{Pr}($ Case 2$)$. Now, to find the PER, the approximation of $Q$ shown in (51) must be used. By noting the change of $\gamma$ subscript, it can be seen that $P_{e_{j}}^{\mathrm{O}, \text { Case } 2}$ follows (55) with $c=1, \rho=\rho_{P}^{\mathrm{N}}$ and $\eta=1$. However, $P_{e_{j}}^{\mathrm{O}, \text { Case } 1}$ is different as the lower and upper integration limits are $\rho_{P}^{\mathrm{O}}$ and $\rho_{P}^{\mathrm{N}}$, respectively. Therefore, the integral in (53) can be adjusted to fit this case as follows,

$$
\begin{aligned}
& I_{3}=\frac{2 \exp \left(\frac{r}{2}+\frac{1}{\bar{\gamma}_{P}}\right)}{\bar{\gamma}_{P} \sqrt{(2 \pi)^{r}}} \\
& \quad \times \int_{\sqrt{\rho_{P}^{\mathrm{O}}+1}}^{\sqrt{\rho_{P}^{\mathrm{N}}+1}} \frac{1}{t^{r-1}} \exp \left(-t^{2}\left[\frac{r}{2}+\frac{1}{\bar{\gamma}_{P}}\right]\right) \mathrm{d} t
\end{aligned}
$$

which can be solved in close-from using [34, p. 109],

$$
\begin{aligned}
I_{3}=\frac{\exp \left(\frac{r}{2}+\frac{1}{\bar{\gamma}_{P}}\right)\left(\frac{r}{2}+\frac{1}{\bar{\gamma}_{P}}\right)^{\frac{r-2}{2}}}{\bar{\gamma}_{P} \sqrt{(2 \pi)^{r}}} \\
\times\left[\Gamma\left(\frac{2-r}{2},\left[\frac{r}{2}+\frac{1}{\bar{\gamma}_{P}}\right]\left(\rho_{P}^{\mathrm{O}}+1\right)\right)-\right. \\
\left.\Gamma\left(\frac{2-r}{2},\left[\frac{r}{2}+\frac{1}{\bar{\gamma}_{P}}\right]\left(\rho_{P}^{\mathrm{N}}+1\right)\right)\right] .
\end{aligned}
$$

Therefore, $P_{e_{P}}^{\mathrm{O}, \text { Case } 1}$ can be found at the top of next page. On the other hand, $P_{e_{S}}^{\mathrm{O}}$ follows (55) with $c=1, \rho=\rho_{S}^{\mathrm{O}}$ and $\eta=1$.

\section{B. NOMA PER}

The PER of NOMA would be intractable if the instantaneous BER shown in (6) and (7) are being used as they are in the form of a sum of $Q$ terms. Therefore, it is proposed to approximate these expressions considering the dominant $Q$ term. Hence, the approximated instantaneous BER for NOMA system can be written as

$$
P_{B_{P}}^{\mathrm{N}} \approx \frac{1}{2} Q\left(\sqrt{c_{1, P}^{2} \gamma_{P}}\right)
$$

and

$$
P_{B_{S}}^{\mathrm{N}} \approx v Q\left(\sqrt{c_{\min , S}^{2} \gamma_{S}}\right)
$$

where $c_{\min , S}=\min \left(c_{1, S}, c_{3, S}\right), v=1$ if $c_{\min , S}=c_{1, S}$ or $v=1 / 2$ if $c_{\min , S}=c_{3, S}$. Therefore, $P_{e_{P}}^{\mathrm{N}}$ follows (55) with $c=c_{1, P}, \rho=\rho_{P}^{\mathrm{N}}$ and $\eta=1 / 2$, where the change to $\gamma$ subscript should be taken in consideration. Similarly, $P_{e_{S}}^{\mathrm{N}}$ follows (55) with $c=c_{\min , S}, \rho=\rho_{S}^{\mathrm{N}}$ and $\eta=v$.
TABLE II: Simulation Parameters and Assumptions.

\begin{tabular}{|c|c|}
\hline \multicolumn{1}{|c|}{ Parameter } & Value \\
\hline \hline Modulation scheme & BPSK \\
\hline Pathloss exponent & $\lambda=2.7$ \\
\hline Distance from BS & $\Upsilon_{P}=1.67 d, \Upsilon_{S}=d$ \\
\hline Channel Model & Block fading \\
\hline Block Length & $L=32$ \\
\hline No. of Drops & $300 \times 10^{3}$ \\
\hline BER QoS Constraint & $\tau_{P}=\tau_{S}=10^{-2}[36]$ \\
\hline FEC & - \\
\hline Address field & Perfect \\
\hline Classifier & Imperfect \\
\hline CSIR and CSIT & Perfect \\
\hline
\end{tabular}

\section{Throughrut AnAlysis}

The user's throughput, $R_{j}$, is the fraction of the transmitted packets that are delivered error-free to the receiver. Therefore, it is fundamental to account for the imperfection caused by the receiver. For instance, the PU throughput will depend on the PER only, whereas the throughput of the SU will depend on the PER as well as the errors caused by the classifier and counting rules. In addition, the probabilities of the TMs are crucial to finding the users' throughput and system's throughput.

The PU throughput highly depends on the probability of $\mathrm{TM}_{1}$ and $\mathrm{TM}_{2}$ besides $P_{e_{P}}^{m}$. Therefore, it can be expressed by

$$
R_{P}=\operatorname{Pr}\left(\mathrm{TM}_{1}\right) \times\left(1-P_{e_{P}}^{\mathrm{O}}\right)+\operatorname{Pr}\left(\mathrm{TM}_{2}\right) \times\left(1-P_{e_{P}}^{\mathrm{N}}\right) .
$$

On the other hand, while assuming that the throughput is zero when the classification error occurs [28], the SU throughput can be written as

$$
\begin{aligned}
R_{S} & =\operatorname{Pr}\left(\mathrm{TM}_{2}\right) \times\left(1-P_{\mathrm{M}}\right) \times\left(1-P_{e_{S}}^{\mathrm{N}}\right) \\
& +\operatorname{Pr}\left(\mathrm{TM}_{3}\right) \times\left(1-P_{\mathrm{FA}_{2}}\right) \times\left(1-P_{e_{S}}^{\mathrm{O}}\right) .
\end{aligned}
$$

It is noted that besides $P_{e_{S}}^{m}$, the $R_{S}$ is affected by the imperfections of the classifier and the counting rule. Finally, the system's throughput can be found by summing the users' throughput, i.e. $R=R_{P}+R_{S}$.

\section{NumERICAL RESUlts AND Discussion}

This section presents the analytical and numerical results of the imperfect receivers as well as the system's throughput, where the main performance trends are highlighted and discussed. In addition, the proposed system's throughput is benchmarked with the conventional NOMA system. To unify the counting rules with respect to the $K$-out-of- $L$ rule, the OR rule is denoted with $K=1 / L$, the Majority rule is denoted with $K=1 / 2$ and the AND rule is denoted with $K=1$. Unless otherwise stated, $K=7 / 8$ for the $K$-out-of- $L$ rule. Also, the power coefficients are fixed to $\alpha_{S}=0.1$ and $\alpha_{P}=0.9$ for the PU/SU-NOMA transmission unless otherwise stated. In addition, $n=3$ for the $Q$ function approximation unless otherwise stated. Table II summarizes the simulation parameters and main assumptions. It is important to mention that where applicable, the simulation results are presented in the figures using markers whereas the analytical results are represented by the lines.

Fig. 3 shows the analytical and simulated classifier performance for a local observation. The figure shows that the simulation and analytical results are matched for all SNR and various 


$$
\begin{aligned}
& P_{e_{P}}^{\mathrm{O}, \text { Case } 1}=1-\frac{1}{F_{\gamma_{P}}\left(\rho_{P}^{\mathrm{N}}\right)-F_{\gamma_{P}}\left(\rho_{P}^{\mathrm{O}}\right)} \sum_{r=0}^{L}\left(\begin{array}{c}
L \\
r
\end{array}\right)(-1)^{r} \frac{\exp \left(\frac{r}{2}+\frac{1}{\bar{\gamma}_{P}}\right)\left(\frac{r}{2}+\frac{1}{\bar{\gamma}_{P}}\right)^{\frac{r-2}{2}}}{\bar{\gamma}_{P} \sqrt{(2 \pi)^{r}}} \\
& \times\left[\Gamma\left(\frac{2-r}{2},\left[\frac{r}{2}+\frac{1}{\bar{\gamma}_{P}}\right]\left(\rho_{P}^{\mathrm{O}}+1\right)\right)-\Gamma\left(\frac{2-r}{2},\left[\frac{r}{2}+\frac{1}{\bar{\gamma}_{P}}\right]\left(\rho_{P}^{\mathrm{N}}+1\right)\right)\right] .
\end{aligned}
$$

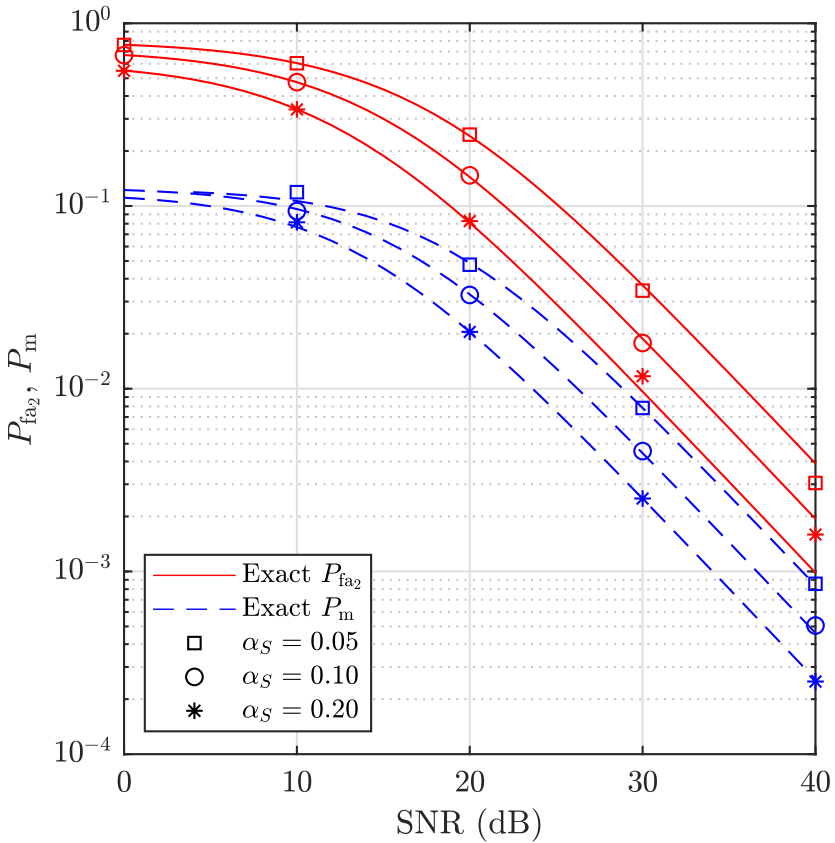

Fig. 3: Analytical and simulated classifier performance versus SNR.

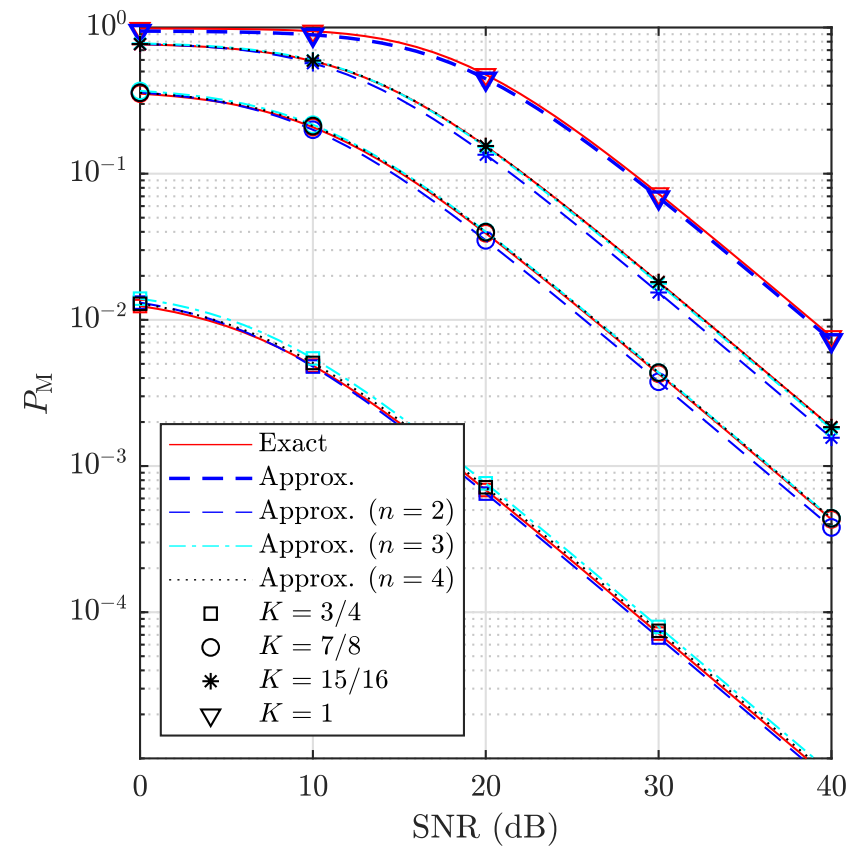

Fig. 4: Exact and approximate global probability of missdetection versus SNR.

$\alpha_{S}$ values. Additionally, it is shown that $P_{\mathrm{m}}$ has a lower value compared to $P_{\mathrm{fa}_{2}}$ which is expected because of the weighting

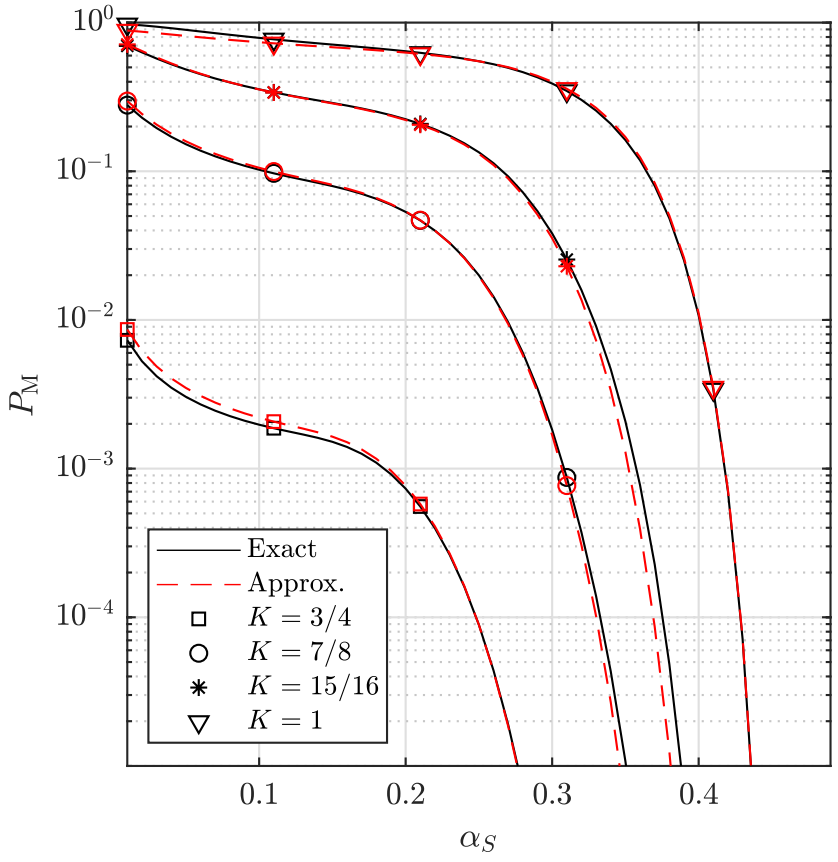

Fig. 5: Exact and approximate global probability of missdetection versus $\alpha_{S}$ for $\mathrm{SNR}=15 \mathrm{~dB}$.

factor $1 / 2$ that appears in (49) while it vanishes from (48).

Figs. 4 and 5 show the exact global miss-detection performance using the numerical evaluation of (44) and the approximate expressions derived in Subsec. IV-C. The figures show that the approximation matches closely the actual performance for all ranges of $K$, SNR and $\alpha_{S}$. Fig. 4 shows that as $n$ increases, the approximation error decreases, however, the drawback is the increase of the computational complexity. In the contrary, $n=3$ provides accurate results while maintaining a low computational complexity compared to $n=4$. Additionally, $P_{\mathrm{M}}$ is negligible for $K=1 / L$, and hence, it can be ignored. Interestingly, it is noted that $P_{\mathrm{M}}$ performance can never be worse than the $K=1$ performance which can be seen as an upper bound. Also, as $K \rightarrow 1 / L, P_{\mathrm{M}}$ improves significantly.

Figs. 6 and 7 show the global false alarm performance using the numerical evaluation of (45) and the approximate expressions derived in Subsec. IV-C . The figures show that the approximation is matching the actual performance for all ranges of $K$, SNR and $\alpha_{S}$. Fig. 6 shows that as $n$ increases, the approximation error decreases, however, the drawback is the increase of the computational complexity. On the contrary, $n=3$ provides accurate results while maintaining a low computational complexity compared to $n=4$. Additionally, $P_{\mathrm{FA}_{2}}$ is considered negligible for $K=1$, and hence, it can be ignored. Interestingly, it is noted that $P_{\mathrm{FA}_{2}}$ performance can 


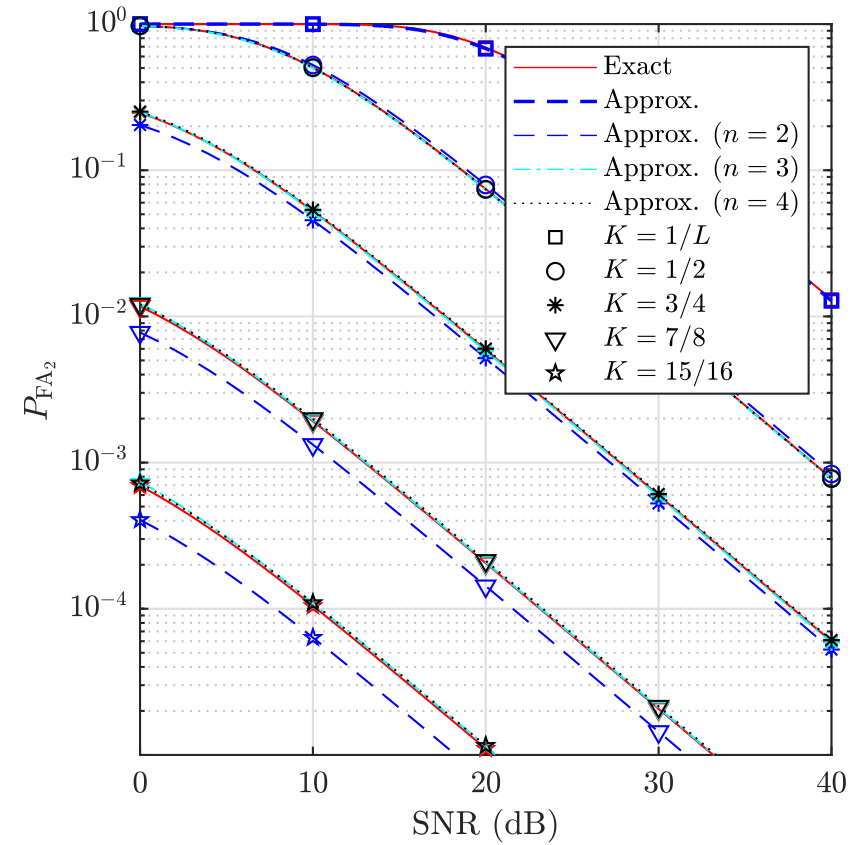

Fig. 6: Exact and approximate global probability of false alarm versus SNR.

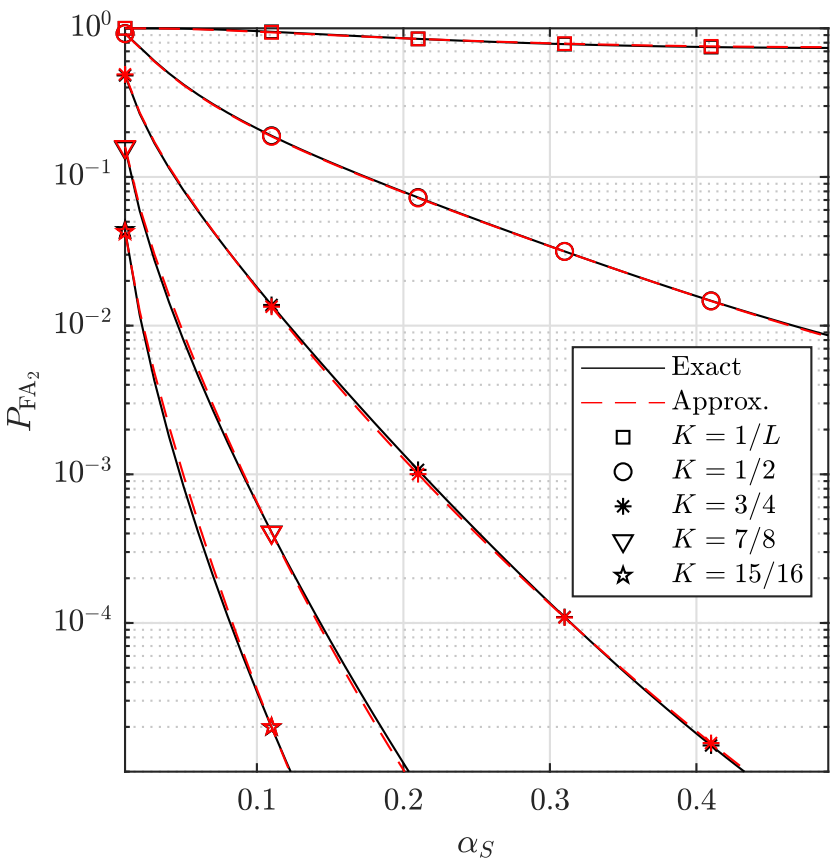

Fig. 7: Exact and approximate global probability of false alarm versus $\alpha_{S}$ for $\mathrm{SNR}=15 \mathrm{~dB}$.

never be worse than the $K=1 / L$ case which can be seen as an upper bound. Also, as $K \rightarrow 1, P_{\mathrm{FA}_{2}}$ improves significantly.

Figs. 8 and 9 show the analytical and simulated PER results of PU and SU for the NOMA and OMA cases. The figure shows the analytical and simulation results match closely for all SNR and $\alpha_{S}$ ranges. Fig. 8 shows that the SU OMA achieves the best PER performance, whereas the PU OMA PER saturates at high SNRs which is justified by the fact that the instantaneous SNR is bounded between two values for one of the cases as seen in (68). Additionally, even though it is an OMA transmission, its performance depends on the selected power coefficients for the NOMA case as $\rho_{P}^{\mathrm{N}}$ is a factor in the PER expression. Further-

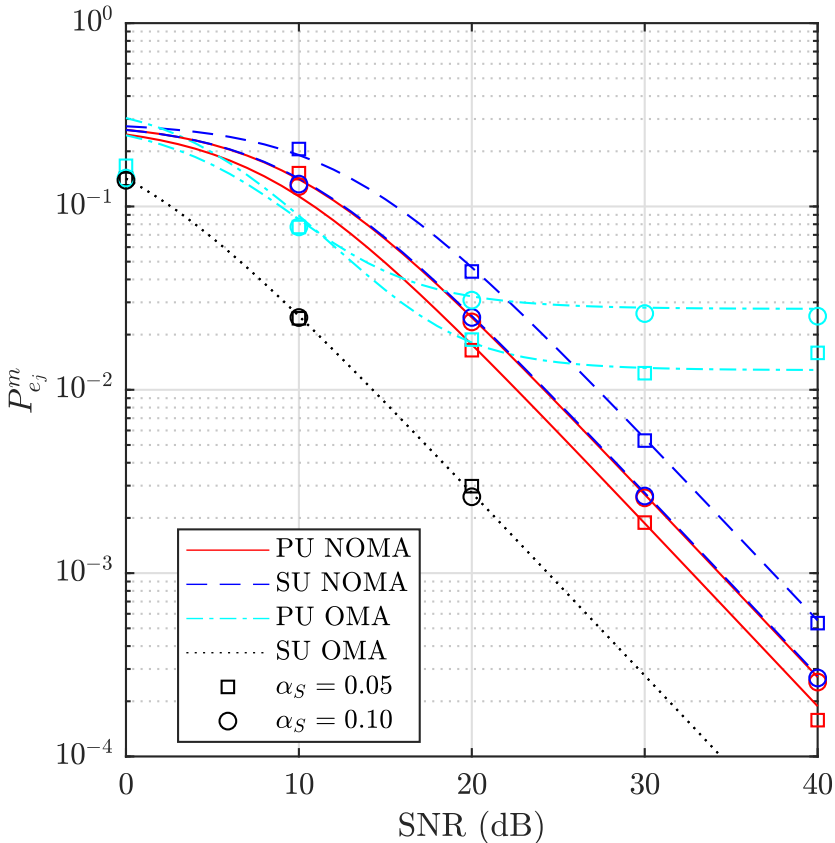

Fig. 8: Analytical and simulated packet error rate versus SNR.

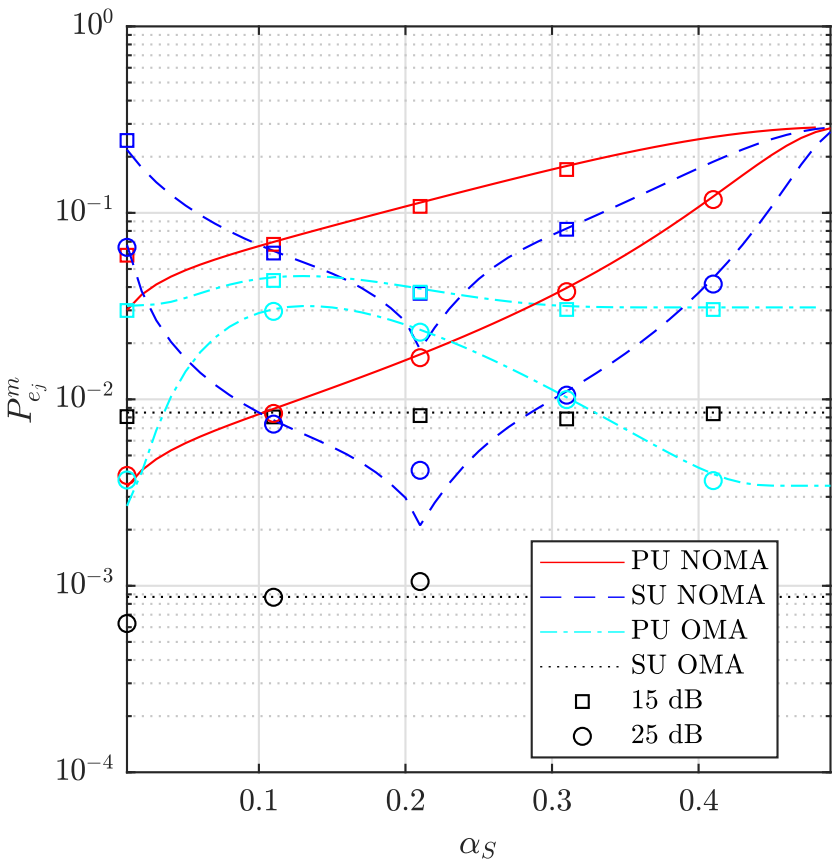

Fig. 9: Analytical and simulated packet error rate performance versus $\alpha_{S}$ for $\mathrm{SNR}=15$ and $25 \mathrm{~dB}$.

more, it is noted from Fig. 9 that the PU NOMA PER degrades as a higher power coefficient is given to the $\mathrm{SU}$ which is aligned with its BER behavior. The case is the same for SU NOMA PER which follows the BER behavior. It is important to highlight that the PER behavior is less sensitive to power allocation compared to $P_{\mathrm{FA}_{2}}$ and $P_{\mathrm{M}}$. Hence, the power allocation should be selected carefully to optimize the $P_{\mathrm{FA}_{2}}$ and $P_{\mathrm{M}}$ as well as the PER.

The analytical and simulated TMs probabilities are shown in Figs. 10 and 11. It is noted from Fig. 10 that at low SNRs, $\operatorname{Pr}\left(\mathrm{TM}_{4}\right)$ is dominating over other TMs because the BER requirement cannot be satisfied for any user. However, as the 


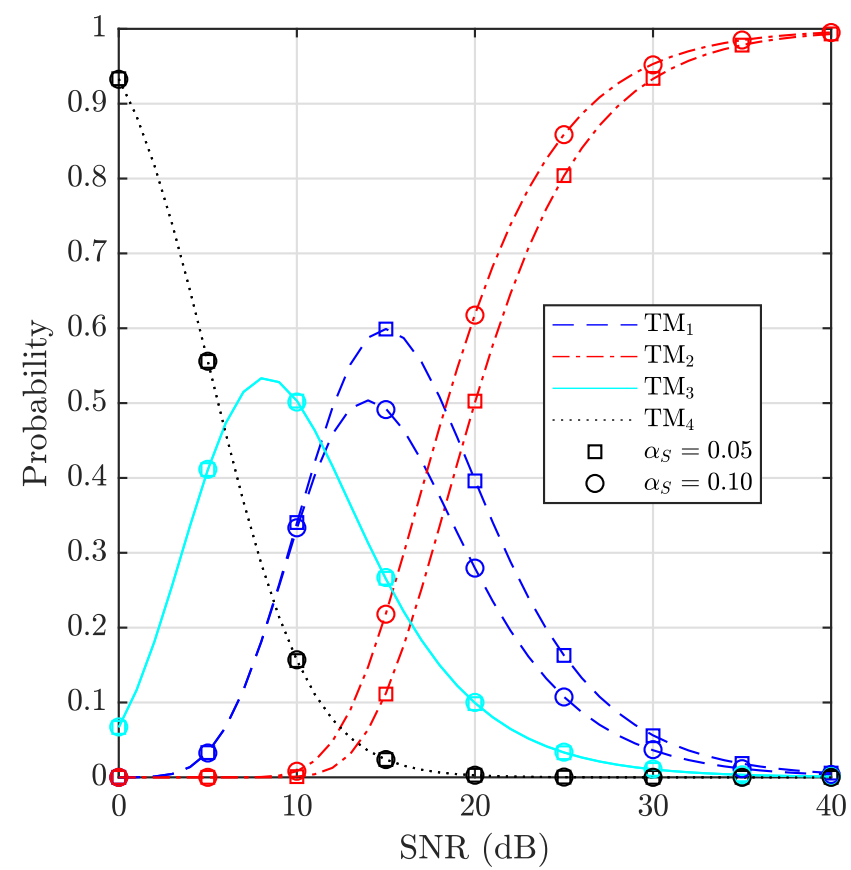

Fig. 10: Analytical and simulated probability of TMs versus SNR.

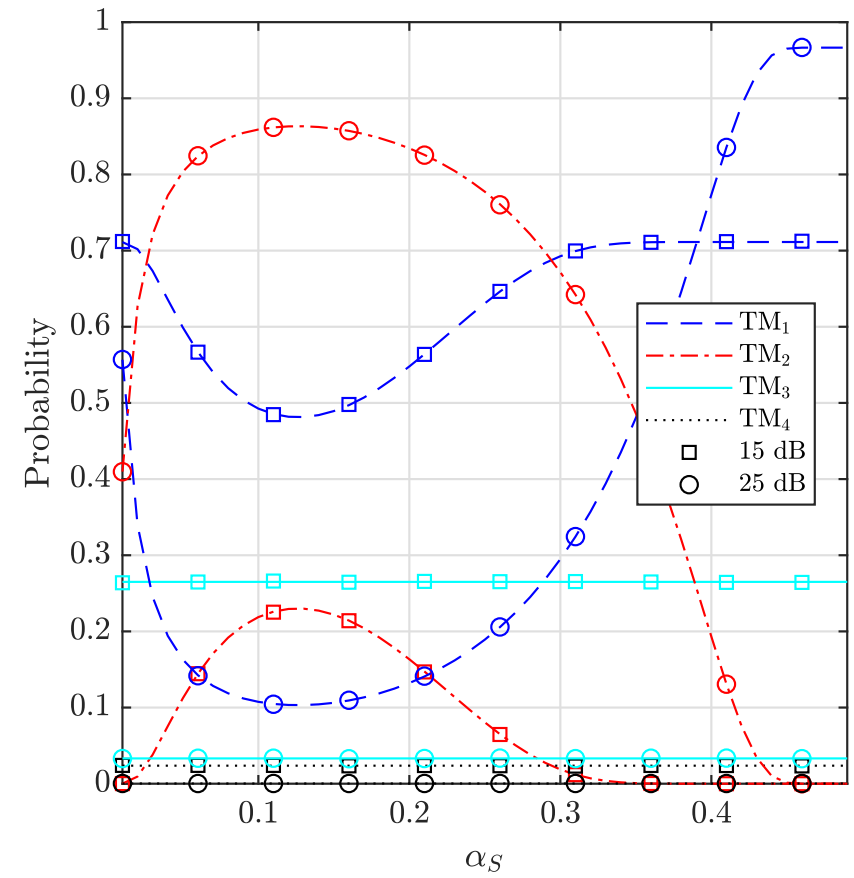

Fig. 11: Analytical and simulated probability of TMs versus $\alpha_{S}$ for $\mathrm{SNR}=15$ and $25 \mathrm{~dB}$.

SNR increases to $6 \mathrm{~dB}, \operatorname{Pr}\left(\mathrm{TM}_{3}\right)$ dominates other TMs because the SU is closer to the base station, and thus, its BER QoS requirement is satisfied with OMA, whereas the PU BER QoS requirement cannot be satisfied with OMA due to the experienced pathloss. As the PU pathloss is compensated for by increasing the $\mathrm{SNR}, \operatorname{Pr}\left(\mathrm{TM}_{1}\right)$ takes over $\operatorname{Pr}\left(\mathrm{TM}_{3}\right)$ because the PU is given higher priority compared to the SU. It can be seen that after about $15 \mathrm{~dB}, \operatorname{Pr}\left(\mathrm{TM}_{2}\right)$ increases and takes over $\operatorname{Pr}\left(\mathrm{TM}_{1}\right)$ as NOMA packets can be sent while satisfying both users' BER QoS requirements. Moreover, Fig. 11 shows that $\operatorname{Pr}\left(\mathrm{TM}_{3}\right)$ and $\operatorname{Pr}\left(\mathrm{TM}_{4}\right)$ are independent from the selected

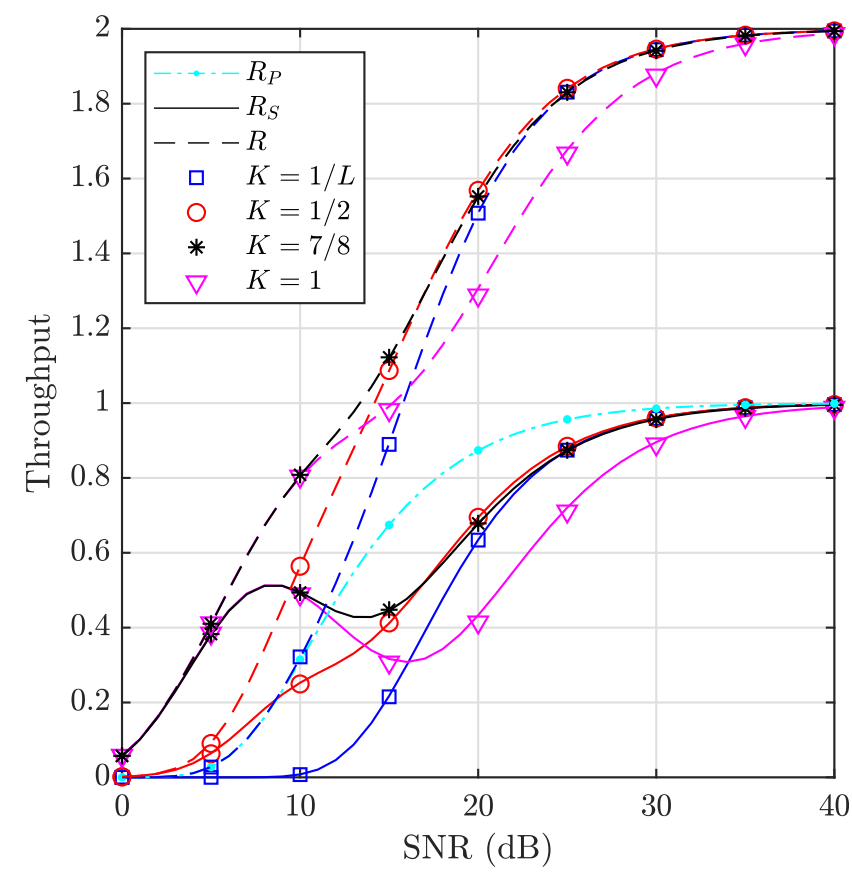

Fig. 12: Analytical and simulated throughput versus SNR.

power coefficients for NOMA mode as seen in (13) and (15). Additionally, it is noted that $\alpha_{S}=0.1$ maximizes $\operatorname{Pr}\left(\mathrm{TM}_{2}\right)$ which is favorable to maximize the system throughput.

Fig. 12 shows the analytical and simulated throughput performance for different $K$ values. It can be seen that optimal $R_{S}$ performance at low SNRs is achieved with $K=1$, whereas $K=1 / L$ achieves an optimal $R_{S}$ performance at high SNRs. For the moderate SNR region, the optimal $R_{S}$ performance is achieved with $K=7 / 8$. This indicates that $K$ should be tuned by the SU based on the average SNR to achieve the optimal performance. Furthermore, by comparing $R_{S}$ to $R_{P}$, it can be seen that $R_{S}$ is higher at low SNRs while $R_{P}$ is better at moderate and high SNRs before saturation.

Finally, Fig. 13 shows the analytical and simulated sum throughput performances for the proposed and conventional systems with the perfect receivers for bench-marking. The figure shows that the performance gap between the perfect receivers and imperfect receivers is wider for the proposed system because of the classifier and counting rules imperfections. Nonetheless, the proposed system shows a performance gain over the conventional system for all SNRs before saturation where the performance gain reduces as the SNR improves. For instance, the performance gain is $\sim 10-13 \mathrm{~dB}$ at 0.4 bit while it reduces to $\sim 7-9 \mathrm{~dB}$ at 0.8 bit. In addition, this figure confirms that $\alpha_{S}=0.1$ maximizes the sum throughput as shown in Fig. 11.

\section{CONCLUSIONS}

To conclude, this article analyzed an uncoordinated hybrid interweave-underlay CR-NOMA system's throughput in Rayleigh block fading channels. The TMs probabilities were derived analytically as well as the PER performance. Also, the classifier and counting rules performances were presented in closed forms. When the proposed system was bench-marked against the conventional NOMA system, the former showed 


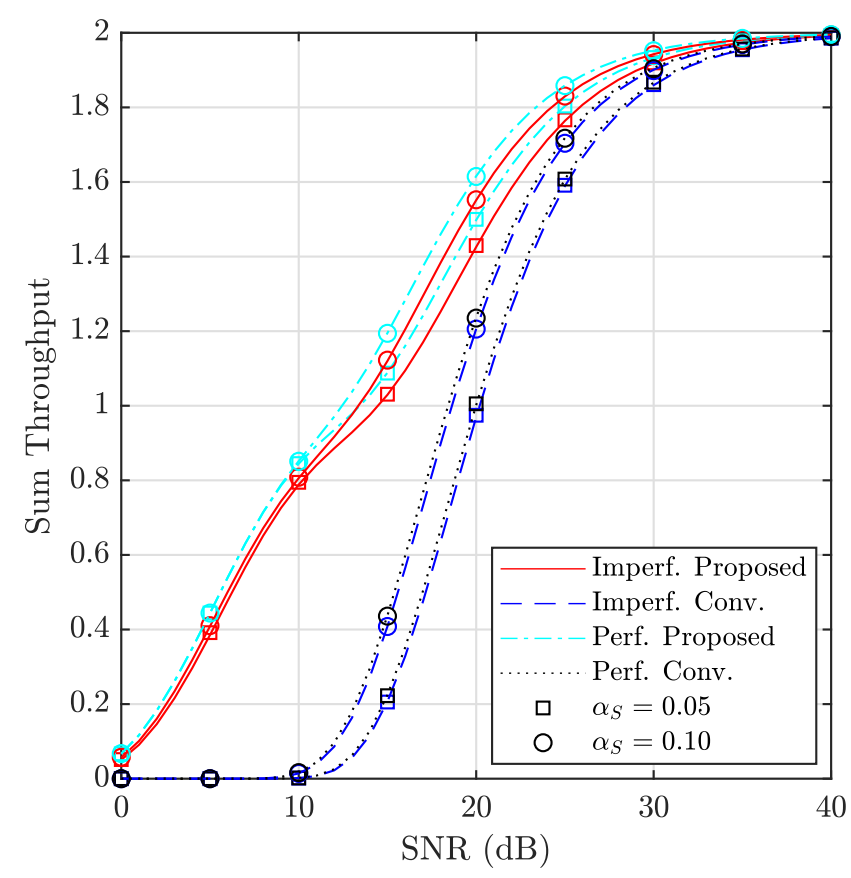

Fig. 13: Analytical and simulated sum throughput versus SNR.

up to $10 \mathrm{~dB}$ performance gain for some scenarios. The SU performance is limited by the classifier and counting rule performance where the latter has to be finely tuned to get an optimal throughput performance. Fortunately, this depends on the average SNR only which can be acquired easily.

\section{REFERENCES}

[1] Y. Liu et al., "Nonorthogonal multiple access for 5G and beyond," Proc. IEEE, vol. 105, no. 12, pp. 2347-2381, Dec. 2017.

[2] F. Kara and H. Kaya, "BER performances of downlink and uplink NOMA in the presence of SIC errors over fading channels," IET Commun., vol. 12, no. 15, pp. 1834-1844, Sept. 2018.

[3] H. Yahya, A. Al-Dweik, and E. Alsusa, "Power-tolerant NOMA using data-aware adaptive power assignment for IoT systems," IEEE Internet of Things J., pp. 1-1, 2021.

[4] T. Assaf, A. Al-Dweik, M. E. Moursi, and H. Zeineldin, "Exact BER performance analysis for downlink NOMA systems over Nakagami- $m$ fading channels," IEEE Access, vol. 7, pp. 134 539-134 555, Sep. 2019.

[5] F. Zhou et al., "State of the art, taxonomy, and open issues on cognitive radio networks with NOMA," IEEE Wireless Commun., vol. 25, no. 2, pp. 100-108, Aprl. 2018.

[6] R. Struzak, "Cognitive radio, spectrum, and evolutionary heuristics," IEEE Commun. Mag., vol. 56, no. 6, pp. 166-171, June 2018.

[7] H. Zhao et al., "Multi-channel cognitive radio ad hoc networks," IEEE Commun. Mag., vol. 56, no. 4, pp. 134-135, Apr. 2018.

[8] T. Yucek and H. Arslan, "A survey of spectrum sensing algorithms for cognitive radio applications," IEEE Commun. Surveys Tuts., vol. 11, no. 1, pp. 116-130, 1st Quart. 2009.

[9] L. Lv, J. Chen, Q. Ni, Z. Ding, and H. Jiang, "Cognitive non-orthogonal multiple access with cooperative relaying: A new wireless frontier for 5G spectrum sharing," IEEE Commun. Mag., vol. 56, no. 4, pp. 188-195, Apr. 2018.

[10] Y. Liu, Z. Ding, M. Elkashlan, and J. Yuan, "Nonorthogonal multiple access in large-scale underlay cognitive radio networks," IEEE Trans. Veh. Technol., vol. 65, no. 12, pp. $10152-10$ 157, Dec. 2016.

[11] S. Arzykulov, G. Nauryzbayev, T. A. Tsiftsis, B. Maham, and M. Abdallah, "On the outage of underlay CR-NOMA networks with detectand-forward relaying," IEEE Trans. Cogn. Commun. Netw., vol. 5, no. 3, pp. 795-804, Sept. 2019.

[12] L. Luo, Q. Li, and J. Cheng, "Performance analysis of overlay cognitive NOMA systems with imperfect successive interference cancellation," IEEE Trans. Commun., vol. 68, no. 8, pp. 4709-4722, May 2020.

[13] Z. Ding, P. Fan, and H. V. Poor, "Impact of user pairing on 5G nonorthogonal multiple-access downlink transmissions," IEEE Trans. Veh. Technol., vol. 65, no. 8, pp. 6010-6023, Aug. 2016.
[14] Z. Yang, Z. Ding, P. Fan, and Z. Ma, "Outage performance for dynamic power allocation in hybrid non-orthogonal multiple access systems," IEEE Commun. Lett., vol. 20, no. 8, pp. 1695-1698, Aug. 2016.

[15] Z. Yang, Z. Ding, P. Fan, and N. Al-Dhahir, "A general power allocation scheme to guarantee quality of service in downlink and uplink NOMA systems," IEEE Trans. Wireless Commun., vol. 15, no. 11, pp. 72447257, Nov. 2016

[16] Z. Ding, L. Dai, and H. V. Poor, "MIMO-NOMA design for small packet transmission in the Internet of Things," IEEE Access, vol. 4, pp. 13931405, Aprl. 2016.

[17] Z. Yang, J. A. Hussein, P. Xu, Z. Ding, and Y. Wu, "Power allocation study for non-orthogonal multiple access networks with multicastunicast transmission," IEEE Trans. Wirel. Commun., vol. 17, no. 6, pp. 3588-3599, June 2018.

[18] Z. Ding, Z. Zhao, M. Peng, and H. V. Poor, "On the spectral efficiency and security enhancements of NOMA assisted multicast-unicast streaming," IEEE Trans. Commun., vol. 65, no. 7, pp. 3151-3163, July 2017.

[19] L. Lv, J. Chen, Q. Ni, and Z. Ding, "Design of cooperative nonorthogonal multicast cognitive multiple access for $5 \mathrm{G}$ systems: User scheduling and performance analysis," IEEE Trans. Commun., vol. 65, no. 6, pp. 2641-2656, June 2017.

[20] Z. Chen, Z. Ding, X. Dai, and G. K. Karagiannidis, "On the application of quasi-degradation to MISO-NOMA downlink," IEEE Trans. Signal Process., vol. 64, no. 23, pp. 6174-6189, Dec. 2016.

[21] J. Shi et al., "Energy efficient resource allocation in hybrid nonorthogonal multiple access systems," IEEE Trans. Commun., vol. 67, no. 5, pp. 3496-3511, May 2019.

[22] H. Abumarshoud, H. Alshaer, and H. Haas, "Dynamic multiple access configuration in intelligent Lifi attocellular access points," IEEE Access, vol. 7, pp. 62 126-62 141, May 2019.

[23] S. Luo and K. C. Teh, "Adaptive transmission for cooperative NOMA system with buffer-aided relaying," IEEE Commun. Lett., vol. 21, no. 4 , pp. 937-940, Aprl. 2017.

[24] M. Bashar, K. Cumanan, A. G. Burr, H. Q. Ngo, L. Hanzo, and P. Xiao, "On the performance of cell-free massive MIMO relying on adaptive NOMA/OMA mode-switching," IEEE Trans. Commun., vol. 68, no. 2, pp. 792-810, Feb. 2020.

[25] P. Xu, J. Quan, Z. Yang, G. Chen, and Z. Ding, "Performance analysis of buffer-aided hybrid NOMA/OMA in cooperative uplink system," IEEE Access, vol. 7, pp. 168 759-168773, Nov. 2019.

[26] N. Nomikos, T. Charalambous, D. Vouyioukas, G. K. Karagiannidis, and R. Wichman, "Hybrid NOMA/OMA with buffer-aided relay selection in cooperative networks," IEEE J. Sel. Topics Signal Process., vol. 13, no. 3, pp. 524-537, June 2019.

[27] M. Alkhawatrah, Y. Gong, G. Chen, S. Lambotharan, and J. A. Chambers, "Buffer-aided relay selection for cooperative NOMA in the Internet of Things," IEEE Internet Things J., vol. 6, no. 3, pp. 5722-5731, June 2019.

[28] M. Choi, D. Yoon, and J. Kim, "Blind signal classification for nonorthogonal multiple access in vehicular networks," IEEE Trans. Veh. Technol., vol. 68, no. 10, pp. 9722-9734, Aug. 2019.

[29] M. Naderpour and H. K. Bizaki, "Low overhead NOMA receiver with automatic modulation classification techniques," IET Commun., vol. 14, no. 5, pp. 768-774, Apr. 2020.

[30] M. Choi and J. Kim, "Blind signal classification analysis and impact on user pairing and power allocation in nonorthogonal multiple access," IEEE Access, vol. 8, pp. 100916-100929, June 2020.

[31] N. Zhang, K. Cheng, and G. Kang, "A machine-learning-based blind detection on interference modulation order in NOMA systems," IEEE Commun. Lett., vol. 22, no. 12, pp. 2463-2466, Dec. 2018.

[32] H. Yahya, E. Alsusa, and A. Dweik, "High throughput wireless networks using downlink cognitive NOMA," submitted to IEEE Network Mag., Dec. 2020.

[33] H. Yahya, E. Alsusa, and A. Al-Dweik, "Blind receiver design for downlink Cognitive-Radio NOMA networks," in IEEE Int. Commun. Conf. (ICC) Workshops), Montreal, Canada, June 2021.

[34] I. S. Gradshteyn and I. M. Ryzhik, Table of integrals, series, and products. Academic press, 2014.

[35] I. M. Tanash and T. Riihonen, "Global minimax approximations and bounds for the Gaussian $q$-function by sums of exponentials," IEEE Trans. Commun., vol. 68, no. 10, pp. 6514-6524, Oct. 2020.

[36] S. Doğan, A. Tusha, and H. Arslan, "NOMA with index modulation for uplink URLLC through grant-free access," IEEE J. Sel. Topics Signal Process., vol. 13, no. 6, pp. 1249-1257, Oct. 2019. 